\title{
Vibration Signal Analysis of Journal Bearing Supported Rotor System by Cyclostationarity
}

\author{
Chang Peng and Lin Bo \\ State Key Laboratory of Mechanical Transmission, Chongqing University, 1510 Main Teaching Building, Chongqing, China \\ Correspondence should be addressed to Lin Bo; bolin0001@aliyun.com
}

Received 2 November 2013; Accepted 9 April 2014; Published 12 May 2014

Academic Editor: Valder Steffen Jr.

Copyright ( 2014 C. Peng and L. Bo. This is an open access article distributed under the Creative Commons Attribution License, which permits unrestricted use, distribution, and reproduction in any medium, provided the original work is properly cited.

\begin{abstract}
Cyclostationarity has been widely used as a useful signal processing technique to extract the hidden periodicity of the energy flow of the mechanical vibration signature. However, the conventional cyclostationarity is restricted to analyzing the real-valued signal, which is incapable of processing the constructed complex-valued signal obtained from the journal bearing supported rotor system operating with oil film instability. In this work, the directional cyclostationary parameters, such as directional cyclic mean, directional cyclic autocorrelation, and directional spectral correlation density, are defined based on the principle of directional Wigner distribution. Practical experiment has demonstrated the effectiveness and superiority of the proposed method in the investigation of the instantaneous planar motion of the journal bearing supported rotor system.
\end{abstract}

\section{Introduction}

Journal bearings are one of the most widely used elements in high speed rotary machines and their performance is of great necessity to the normal operation of the rotor systems supported by oil film bearings. As such, plenty of research on journal bearing malfunction analysis has been conducted.

Oil whirl and oil whip are common failure modes of journal bearings induced by oil film instability when the rotating speed of the shaft exceeds the critical speed of the rotor system. Based on the mathematical model of a symmetric rotor supported by one rigid journal bearing and one oil film journal bearing, Muszynska [1,2] mathematically interpreted the dynamic phenomena related to synchronous vibration and self-excited vibration. Relying on a pair of orthogonally installed proximity sensors, the orbit of the shaft centerline is usually monitored to help in analyzing the system operating condition. In addition, Moosavian et al. [3] compared $\mathrm{K}$-nearest neighbor (KNN) and artificial neural network (ANN) for the power spectral density technique based fault diagnosis of main journal bearings of internal combustion (IC) engine under different conditions. Ying et al. [4] studied the system dynamics characteristics of tilting pad journal bearing rotor system operating around natural frequency or on high-speed range by considering the influence of the pad moment of inertia. Dimond et al. [5] reviewed strengths and weaknesses of identification methods for fluid film journal bearing static and dynamic characteristics, particularly the bearing stiffness, damping, and mass coefficients based on measured data of different measurement systems. The developments and trends in improving bearing measurements were also documented. Moreover, Zanarini and Cavallini [6] illustrated detailed experimental results corresponding to oil whirl and oil whip mixed with misalignment, unbalance, and resonance.

In statistics on random processes, cyclostationary processes belonging to the classes of nonstationary processes are defined to represent the correlation features of periodic phenomenon. As such, it is necessary to analyze the cyclostationarity of the modulated vibration radiated by the journal bearing supported system with its components operating periodically. Gardner firstly defined the concept of cyclostationarity and foresaw the application of it. Then Gardner et al. [7] presented a concise review of the literature on cyclostationarity based on the investigation of an extensive bibliography. In addition, Serpedin et al. [8] attempted to provide a detailed classification group which represented a comprehensive list of references on cyclostationarity and its applications. Mccormick and Nandi [9] compared second 
order cyclostationarity with conventional spectral analysis and synchronous averaging in the condition monitoring of rolling element bearing. They found that the former can reveal significant fault features while the latter failed. Antoni [10] presented a tutorial on cyclostationarity, which introduced key concepts on actual mechanical signals and proved the superiority of cyclostationarity applied in machine diagnostics, identification of mechanical systems, and separation of mechanical sources.

Condition monitoring of journal bearing supported rotor systems usually relies on two orthogonally installed proximity sensors. The orbit signature of the center of the journal is then qualitatively analyzed to investigate system operating condition. Southwick $[11,12]$ applied the full spectrum plot, as compared to the half-spectrum, to diagnose diverse rotating machinery malfunctions. They found that using the full spectrum the orbit ellipticity and vibration precession can be determined without being affected by probe orientation. In order to investigate the complex-valued signal of the instantaneous lateral vibration of the mechanical structure, Lee et al. [13] proposed the two-sided directional power spectra complex-valued vibration signals for the diagnosis of cylinder power faults of four-cylinder compression and spark ignition engines. Furthermore, Lee and Han $[14,15]$ proposed the directional Wigner distribution to effectively determine the procession parameters of the planar motion both in timefrequency domain and in order-frequency domain.

Based on the previous introduction, in this work the concept of directional cyclostationarity of complex-valued signal is defined and used to investigate the vibration of a journal bearing supported rotor system which is influenced by oil film instability. The structure of the paper is organized as follows. In Section 2, the theory responding to the first and the second order cyclostationarity of real-valued signal is reviewed. Furthermore, the directional Wigner distribution is introduced. Subsequently, the proposed directional cyclostationarity is represented. In Section 3, the experiments conducted on a test bench are described and the results obtained from analysis compared and discussed using directional cyclostationarity, directional time frequency distribution, and conventional cyclostationarity, respectively. In Section 4, summary discussions and the conclusions are given.

\section{Directional Cyclostationarity}

2.1. Cyclostationarity of Real-Valued Signals. Since cyclostationarity can indicate itself in statistics of any orders determined by the degree of nonlinear operation, there are various statistic parameters illustrated in the time, the frequency, and the cyclic frequency domains [10]. The first and second order of cyclostationarity are firstly reviewed for simplicity but without losing accuracy.

The mean value of a cyclostationary signal (cyclic mean, $\mathrm{CM})$ is defined as

$$
\mathrm{CM}(\alpha)=\sum_{\alpha \in \widetilde{A}} E\left[x(t) \cdot e^{-2 \pi \alpha t}\right]
$$

where $\alpha$ is the cyclic frequency of the signal and the set $\widetilde{A}$ contains all cyclic frequencies. $E[\cdot]=\lim _{T \rightarrow \infty} \int_{T}(\cdot) d t / T$ is the mean operator.

Then the cyclic autocorrelation (CR) representing the energy of the signal can be defined in as

$$
\mathrm{CR}(\alpha, \tau)=\sum_{\alpha \in \widetilde{A}} E\left[x\left(t-\frac{\tau}{2}\right) x\left(t+\frac{\tau}{2}\right) \cdot e^{-2 \pi \alpha t}\right] .
$$

By taking the Fourier transform of CR with respect to time lag $\tau$, the spectral correlation density (SCD) is given by

$$
\operatorname{SCD}(\alpha, f)=\int_{-\infty}^{\infty} \operatorname{CR}(\alpha, \tau) \cdot e^{-2 \pi f \tau} d \tau
$$

Finally by transforming the cyclic frequency $\alpha$ back to the time domain, the Wigner-Ville spectrum (WV) can be obtained in

$$
\mathrm{WV}(t, f)=\sum_{\alpha \in \widetilde{A}} \operatorname{SCD}(\alpha, f) \cdot e^{j 2 \pi \alpha t}
$$

2.2. Directional Wigner Distribution. Lee and Han $[14,15]$ developed a directional Wigner distribution (DWD) by substituting complex-valued signals for the real-valued signals, which could describe the instantaneous planar motion of the system. In their work, the signals $x(t)$ and $y(t)$ obtained from the horizontal and the vertical proximity probes were combined in:

$$
\begin{aligned}
p(t) & =x(t)+j y(t)=p^{f}(t)+p^{b}(t) \\
& =\left|r^{f}\right| e^{j\left(\omega t+\varphi^{f}\right)}+\left|r^{b}\right| e^{j\left(\omega t+\varphi^{b}\right)},
\end{aligned}
$$

where $p^{f}(t)$ and $p^{b}(t)$ denote the forward and backward, respectively, procession of the rotor centerline.

The $p^{f}(t)$ and $p^{b}(t)$ are given by

$$
\begin{aligned}
& p^{f}(t)=\frac{\{p(t)+j \tilde{p}(t)\}}{2}, \\
& p^{b}(t)=\frac{\{p(t)-j \tilde{p}(t)\}}{2},
\end{aligned}
$$

where $\tilde{p}(t)$ is the Hilbert transform of $p(t)$.

Then the auto-DWD is defined in

$$
\begin{aligned}
& W_{p}^{d}(t, \omega) \\
& =\left\{\begin{array}{l}
W_{p^{f}}(t, \omega) \\
W_{p^{b}}(t, \omega)
\end{array}\right. \\
& = \begin{cases}\int_{-\infty}^{\infty} e^{-j \omega \tau} p^{f^{*}}\left(t-\frac{\tau}{2}\right) p^{f}\left(t+\frac{\tau}{2}\right) d \tau, & \text { for } \omega>0 \\
\int_{-\infty}^{\infty} e^{-j \omega \tau} p^{b^{*}}\left(t-\frac{\tau}{2}\right) p^{b}\left(t+\frac{\tau}{2}\right) d \tau, & \text { for } \omega<0,\end{cases}
\end{aligned}
$$

where $W_{p^{f}}(t, \omega)$ and $W_{p^{b}}(t, \omega)$ are, respectively, the forward and the backward terms and $\omega$ is the circular rotating frequency. 


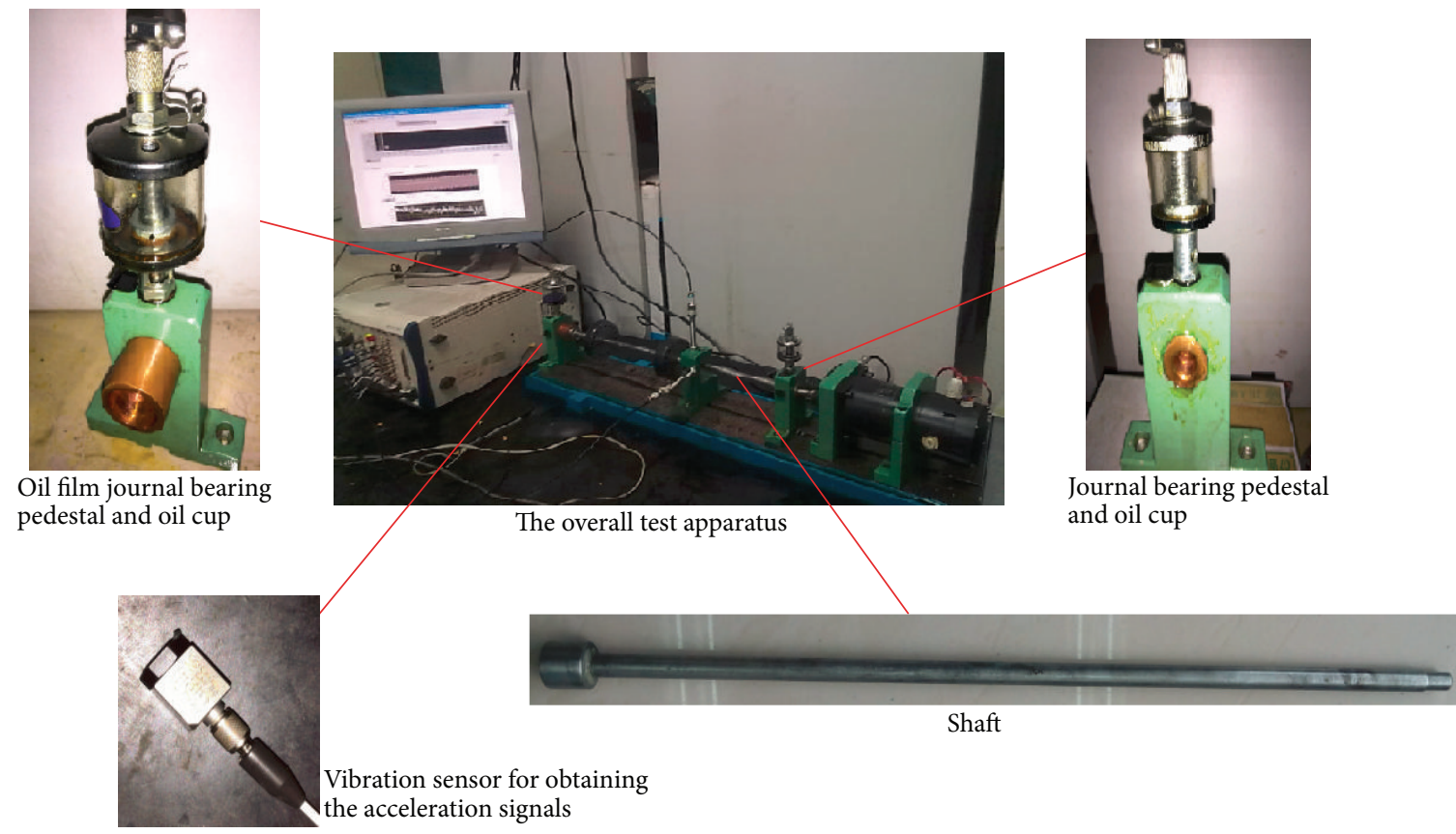

FIGURE 1: Test bench and its main components.

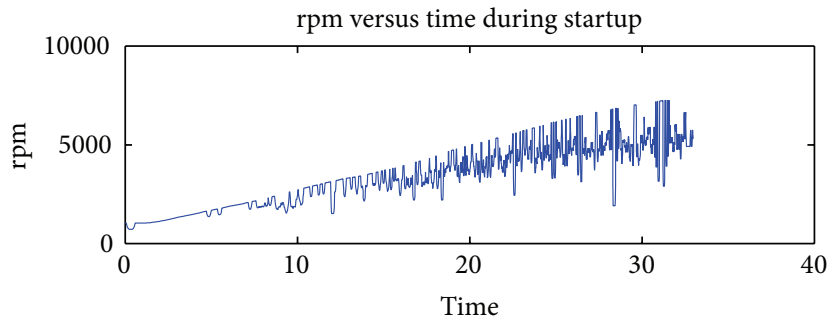

(a)

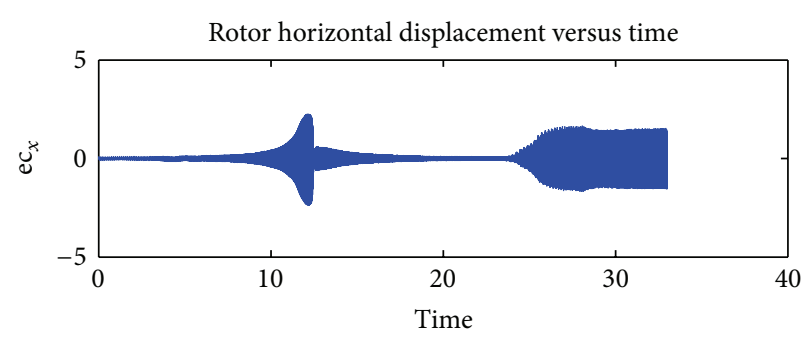

(b)

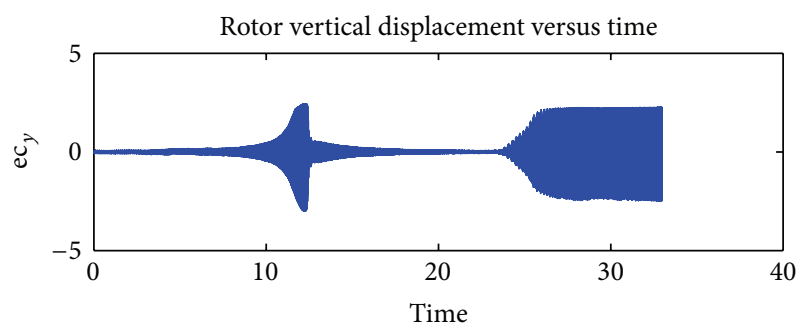

(d)

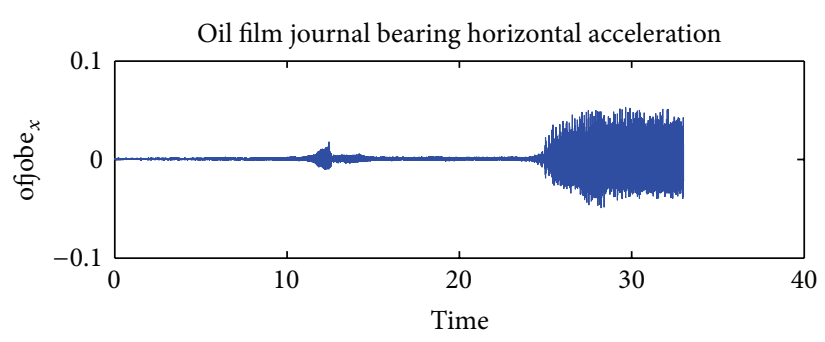

(c)

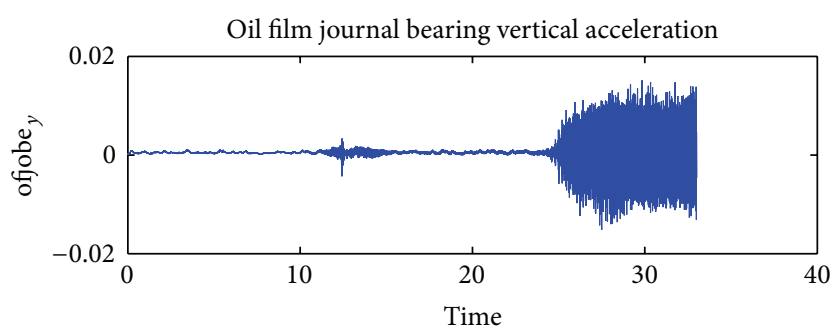

(e)

FIGURE 2: Vibration signal of journal bearing supported system during startup. 


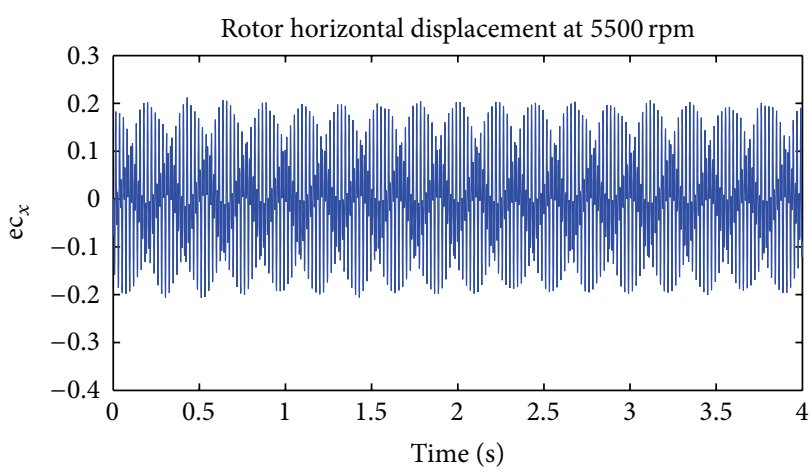

(a)

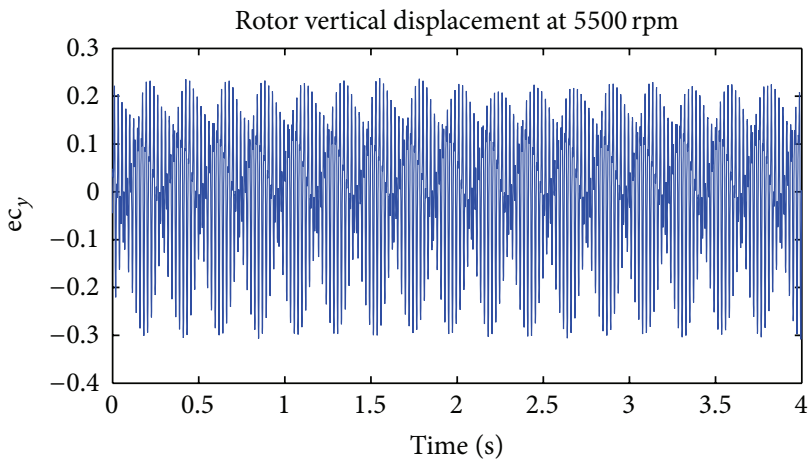

(c)

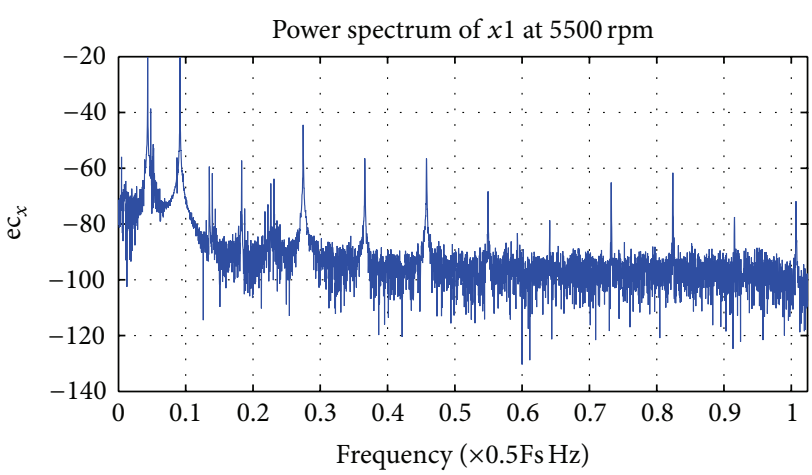

(b)

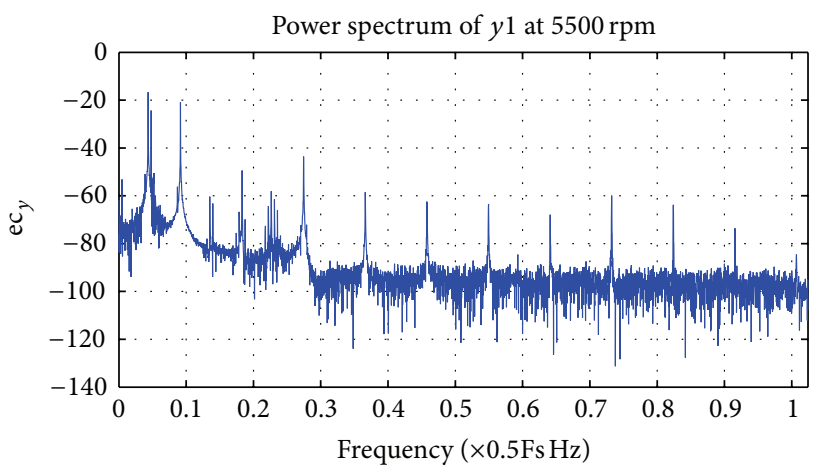

(d)

FIGURE 3: The lateral vibration of the shaft and its power spectrum at $5500 \mathrm{rpm}$.

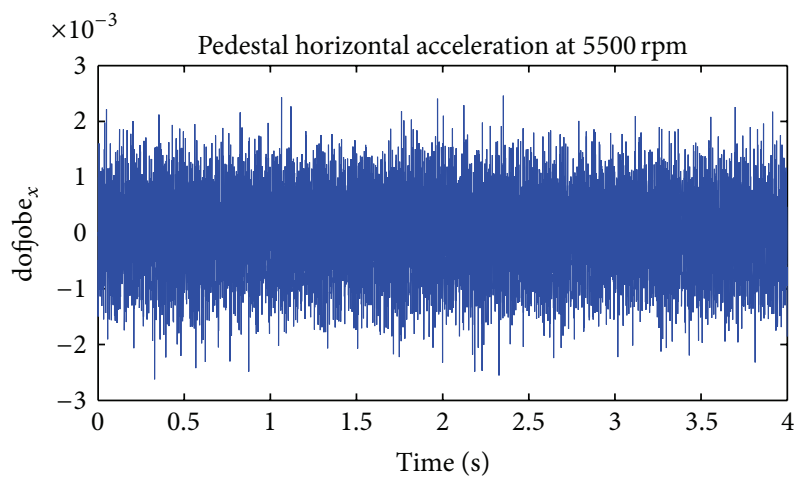

(a)

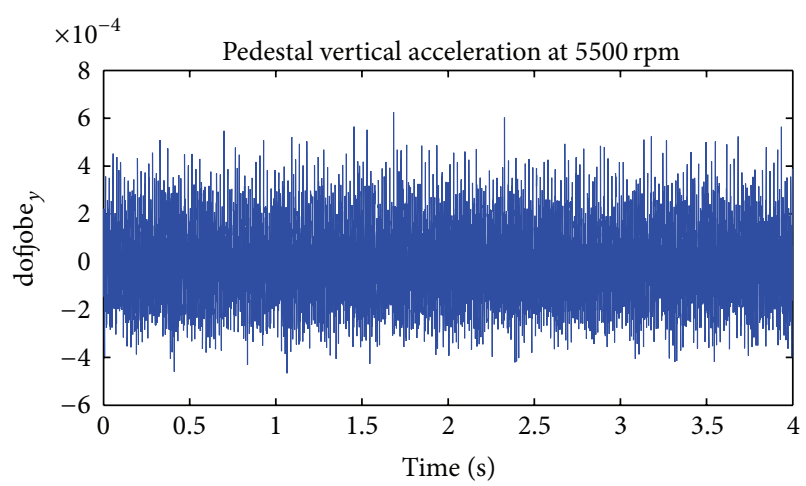

(c)

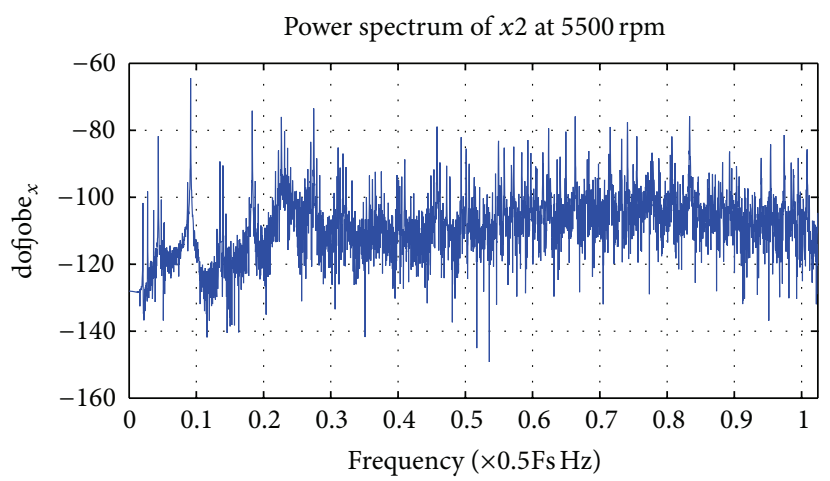

(b)

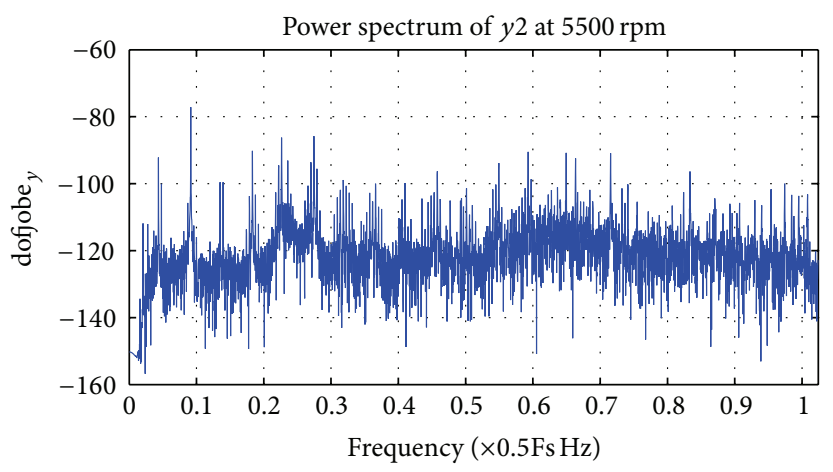

(d)

FIGURE 4: The lateral vibration of the pedestal and its power spectrum at $5500 \mathrm{rpm}$. 


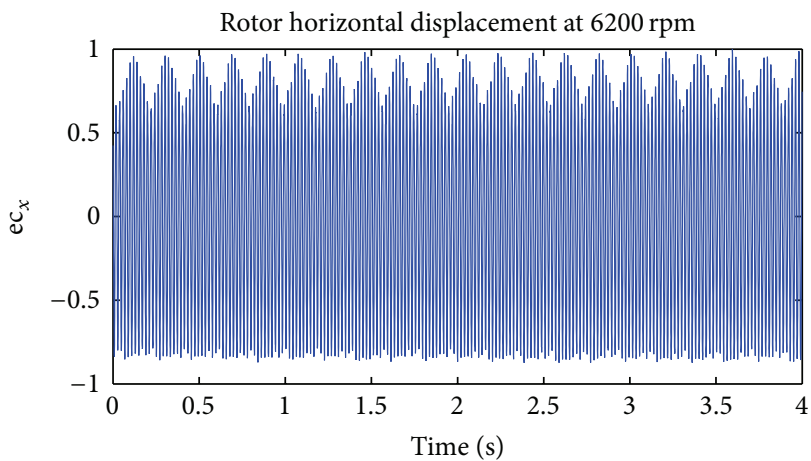

(a)

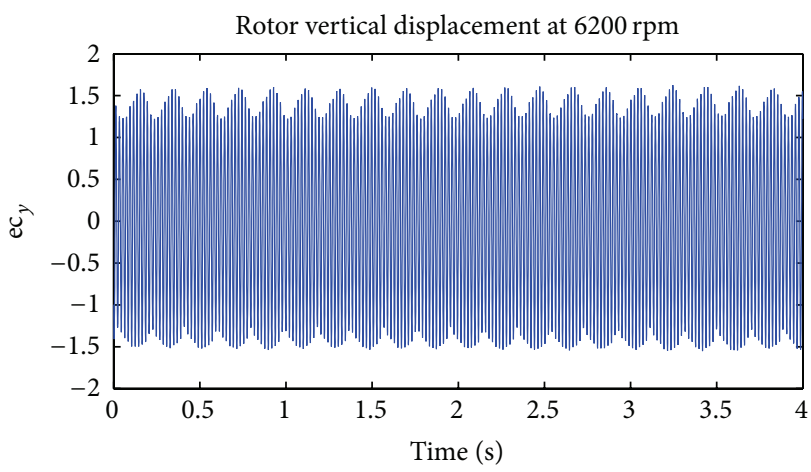

(c)

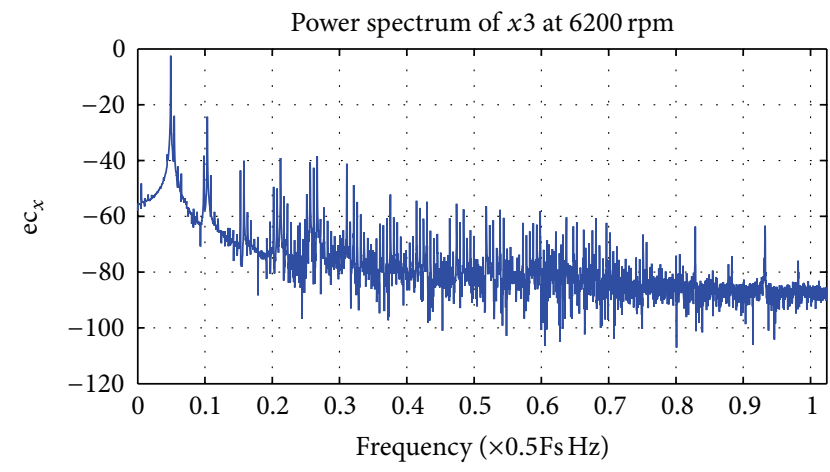

(b)

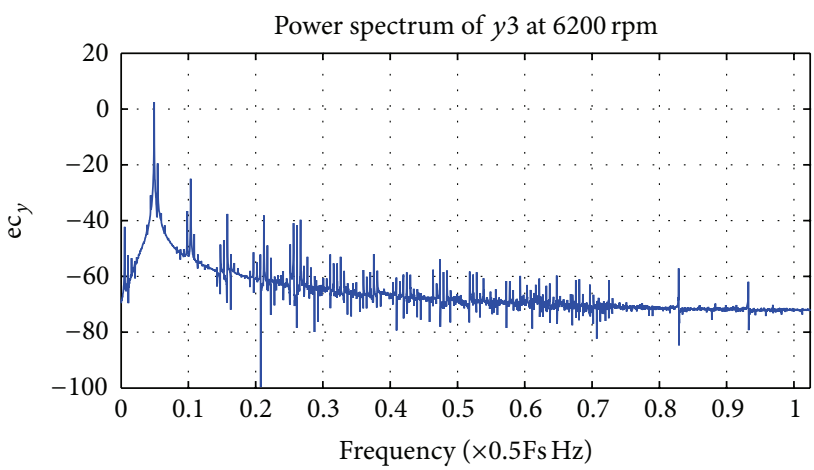

(d)

FIGURE 5: The lateral vibration of the shaft and its power spectrum at $6200 \mathrm{rpm}$.

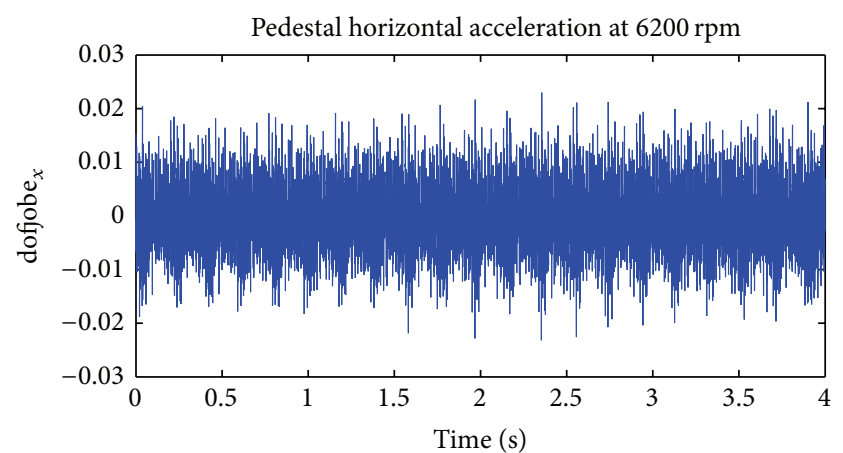

(a)

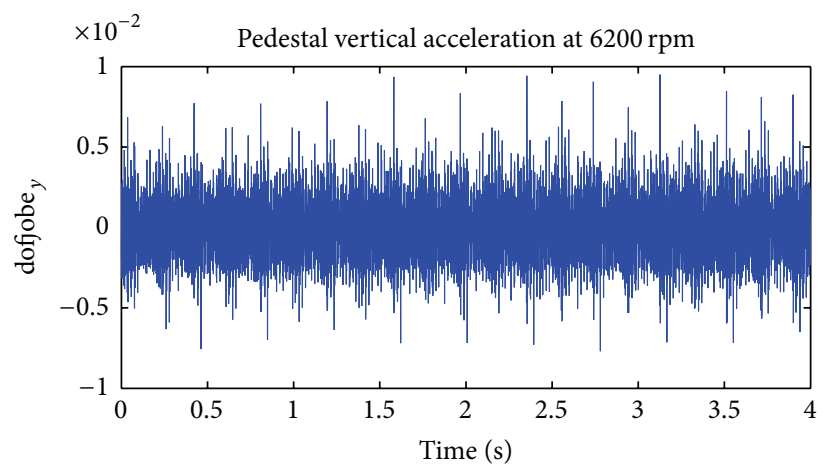

(c)

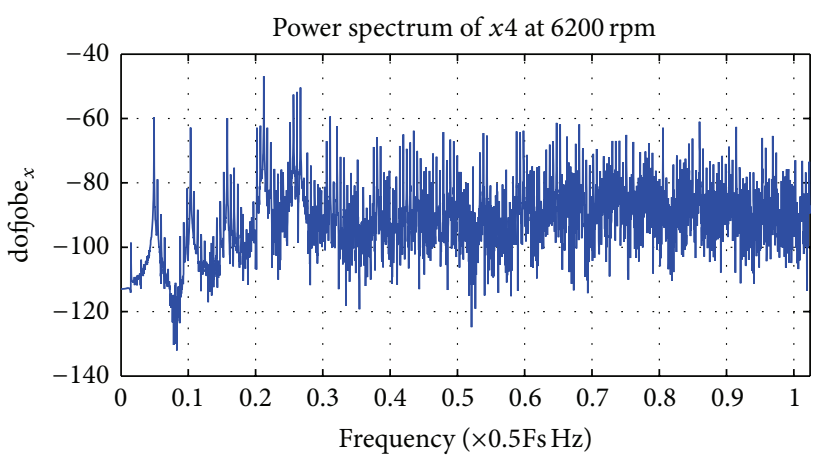

(b)

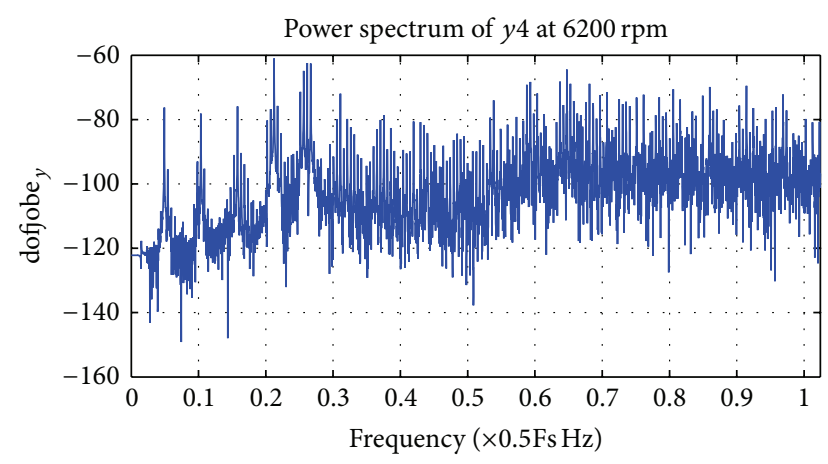

(d)

FIGURE 6: The lateral vibration of the pedestal and its power spectrum at $6200 \mathrm{rpm}$. 


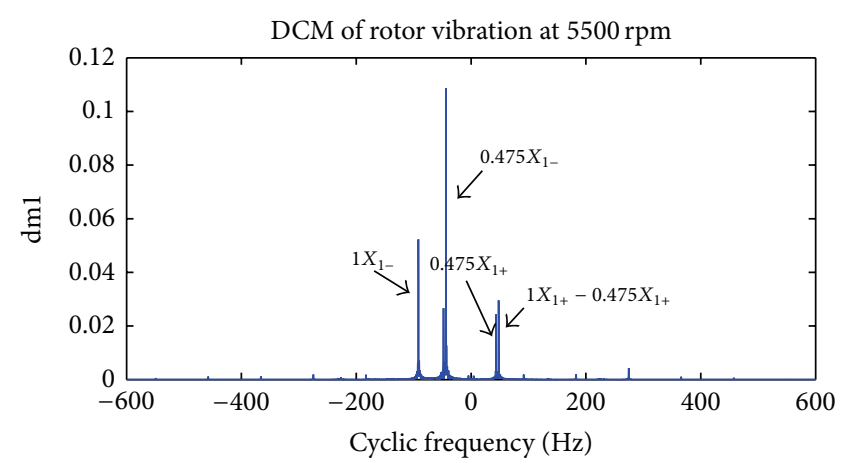

(a)

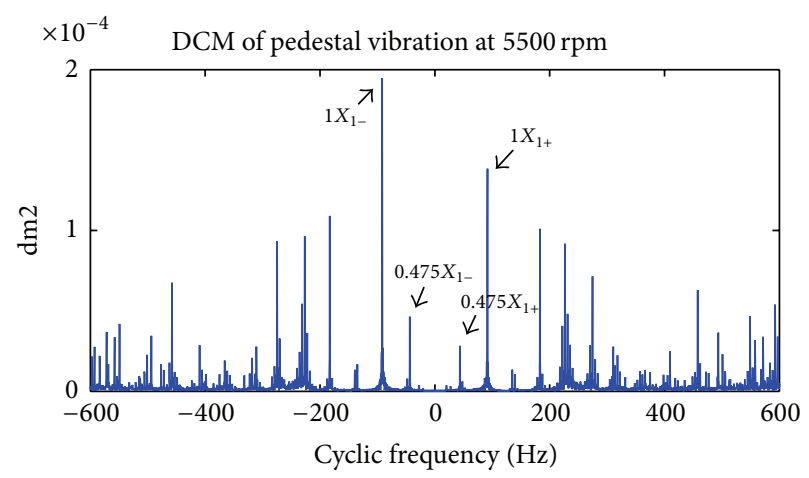

(c)

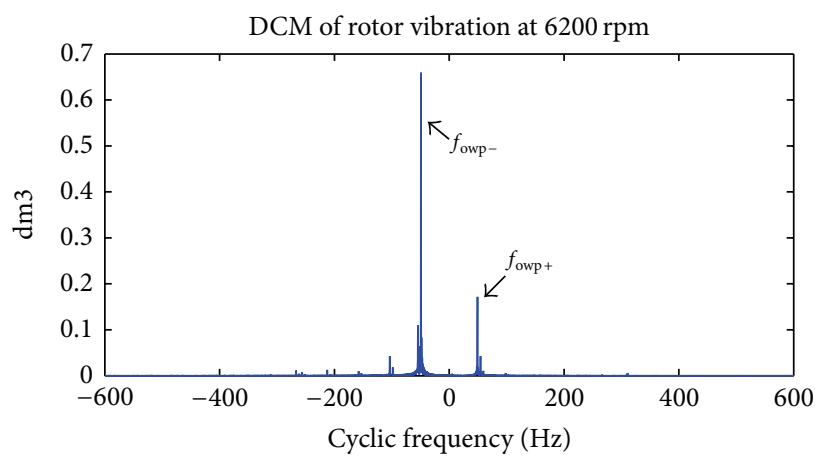

(b)

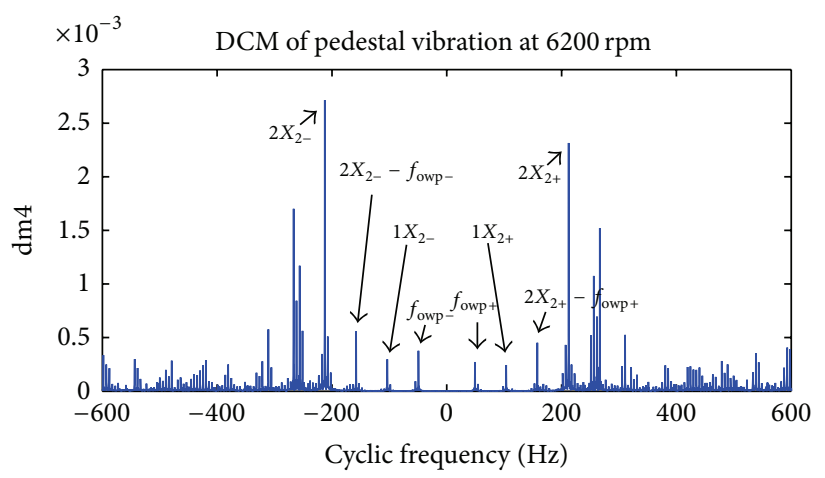

(d)

FIGURE 7: Directional cyclic mean of the lateral vibration of the shaft and pedestal.

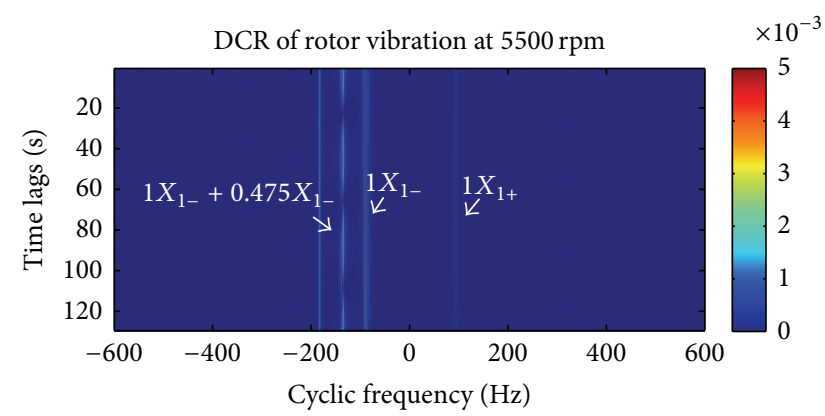

(a)

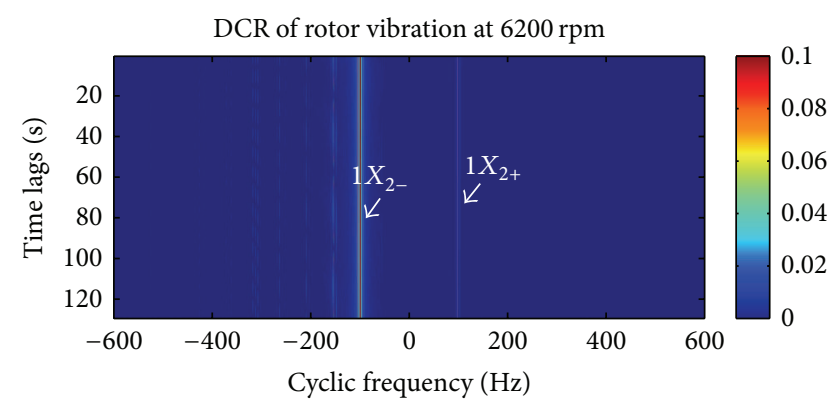

(c)

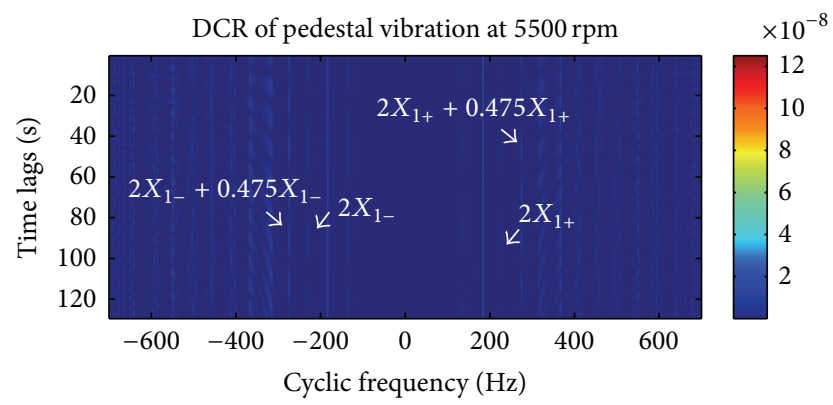

(b)

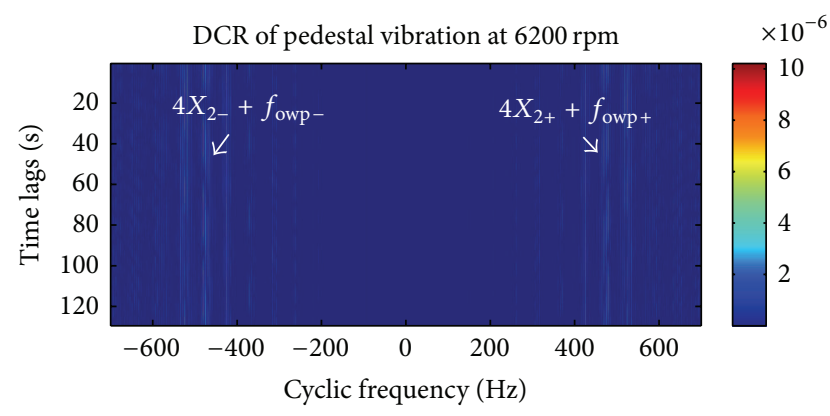

(d)

FIGURE 8: Directional cyclic autocorrelation of the lateral vibration of the shaft and pedestal. 


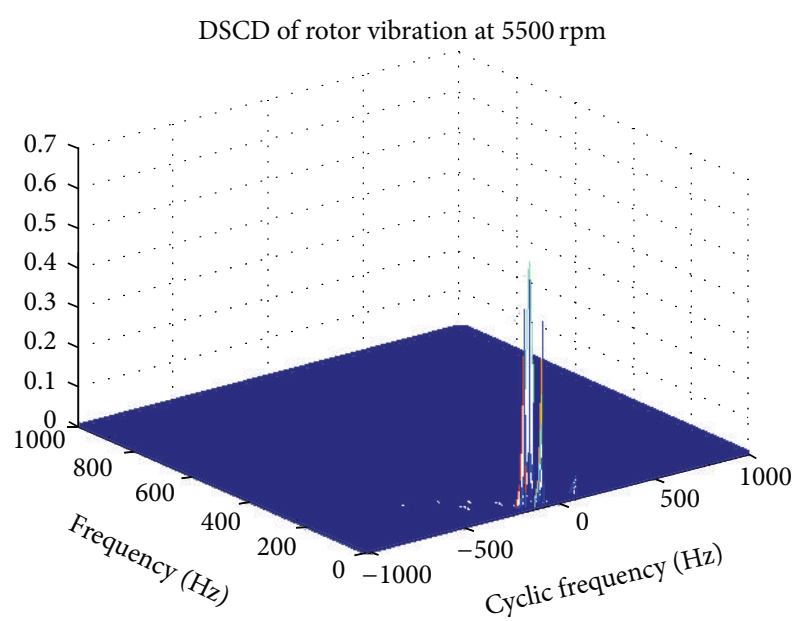

(a)

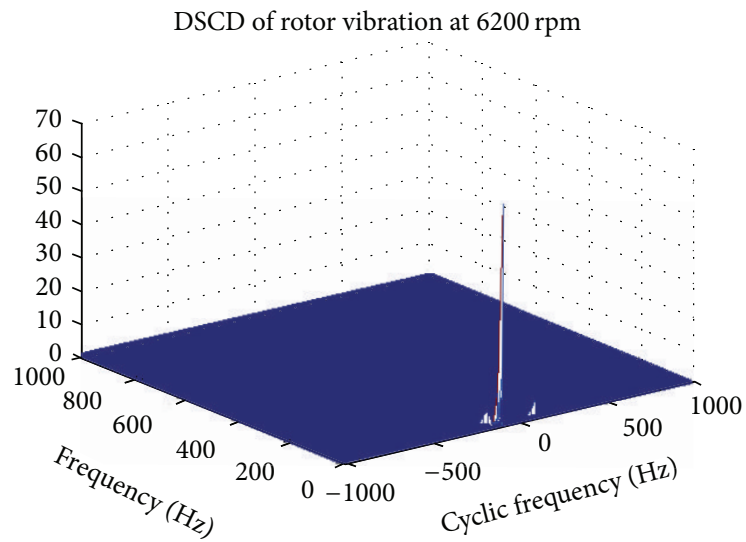

(c)
DSCD of pedestal vibration at $5500 \mathrm{rpm}$

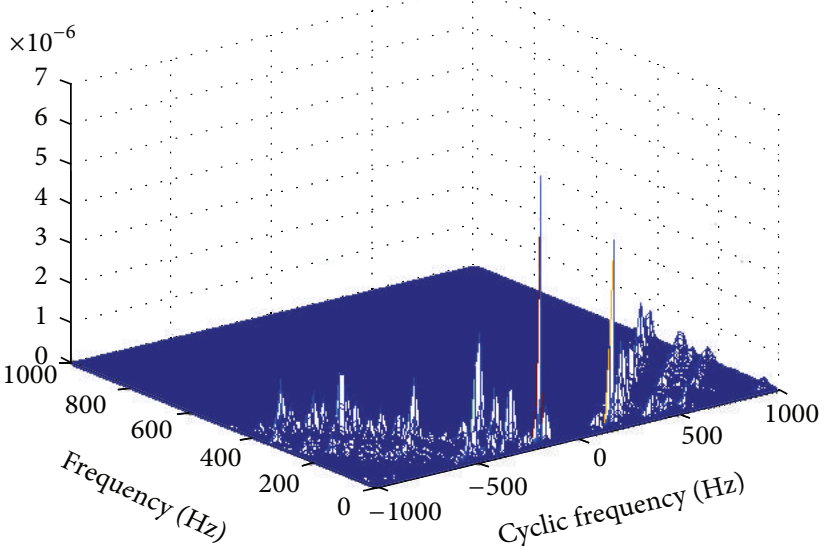

(b)

DSCD of pedestal vibration at $6200 \mathrm{rpm}$

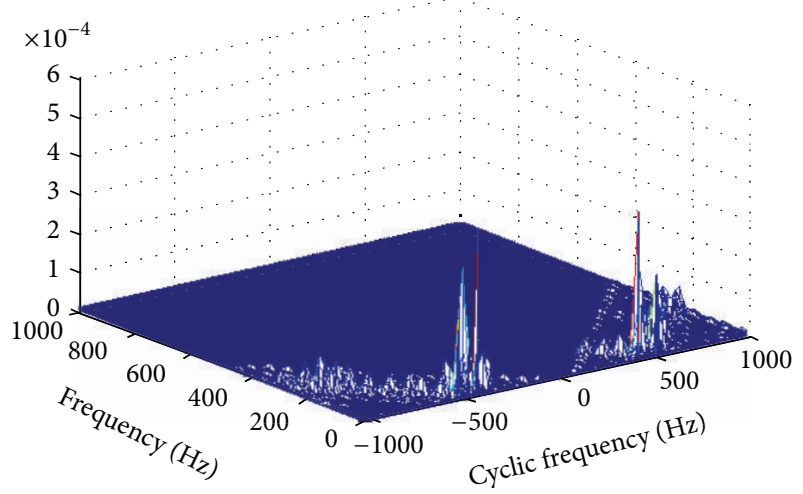

(d)

FIGURE 9: Directional spectral correlation density of the lateral vibration of the shaft and pedestal.

The cross-DWD is given by

$$
\begin{aligned}
W_{p^{*}, p}^{d}(t, \omega) & =W_{p^{b^{*}, p^{f}}}(t, \omega) \\
& =\int_{-\infty}^{\infty} e^{-j \omega \tau} p^{b}\left(t-\frac{\tau}{2}\right) p^{f}\left(t+\frac{\tau}{2}\right) d \tau, \quad \forall \omega .
\end{aligned}
$$

In addition, the full spectrum (FS), another approach for shaft orbit signal analysis, is developed by Southwick [11, 12], defined in

$$
\begin{aligned}
\mathrm{FS} & (t, \omega) \\
& = \begin{cases}\mathrm{FS}_{p^{f}}(t, \omega) \\
\mathrm{FS}_{p^{b}}(t, \omega)\end{cases} \\
& = \begin{cases}\int_{-\infty}^{\infty} e^{-j \omega t} p^{f}(t) d t, & \text { for } \omega>0 \\
\int_{-\infty}^{\infty} e^{-j \omega t} p^{b}(t) d t, & \text { for } \omega<0\end{cases}
\end{aligned}
$$

2.3. Proposed Directional Cyclostationarity of Complex-Valued Signals. Originated from the cyclostationarity for real-valued signal, in this work the directional cyclostationarity for complex-valued vibration signal is developed according to the principle of the previously introduced directional Wigner distribution. The proposed method can be applied not only to characterize the hidden periodicities of the vibration radiated by the journal bearing supported system but also to determine the procession directivity of the planar motion in time-frequency domain by analyzing the combined complexvalued vibration signal.

The directional cyclic mean (DCM) can be defined in (10) according to (1) and (7):

$$
\begin{aligned}
& \operatorname{DCM}(\alpha) \\
& = \begin{cases}\operatorname{DCM}_{f}(\alpha) \\
\operatorname{DCM}_{b}(\alpha)\end{cases} \\
& = \begin{cases}\sum_{\alpha \in \widetilde{A}} E\left[p^{f}(t) \cdot e^{-2 \pi \alpha t}\right], & \text { for } \alpha>0 \\
\sum_{\alpha \in \widetilde{A}} E\left[p^{b}(t) \cdot e^{-2 \pi \alpha t}\right], & \text { for } \alpha<0,\end{cases}
\end{aligned}
$$




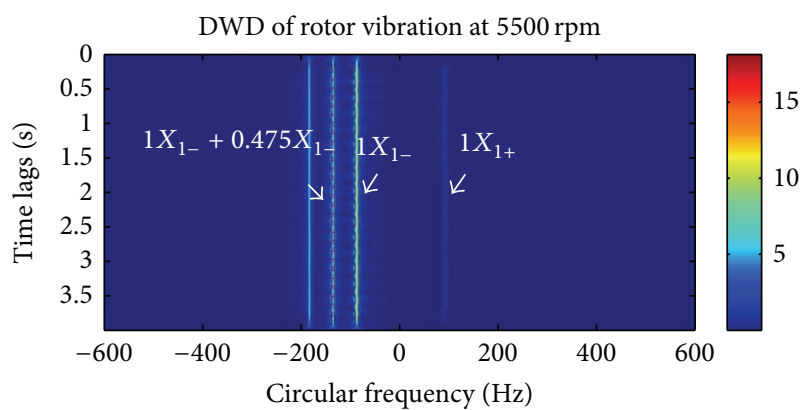

(a)

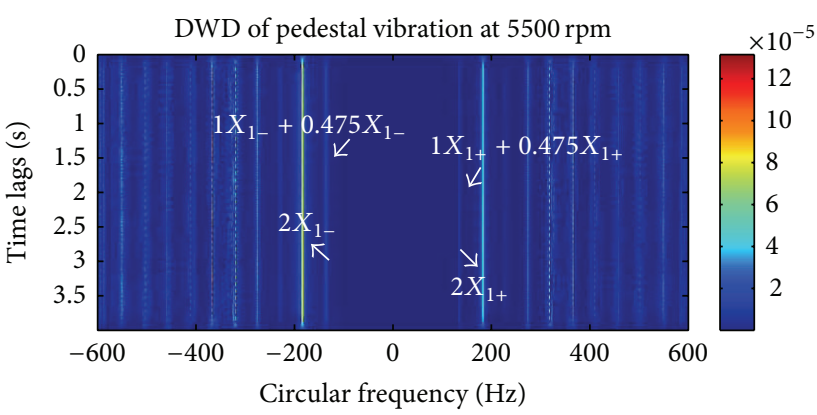

(c)

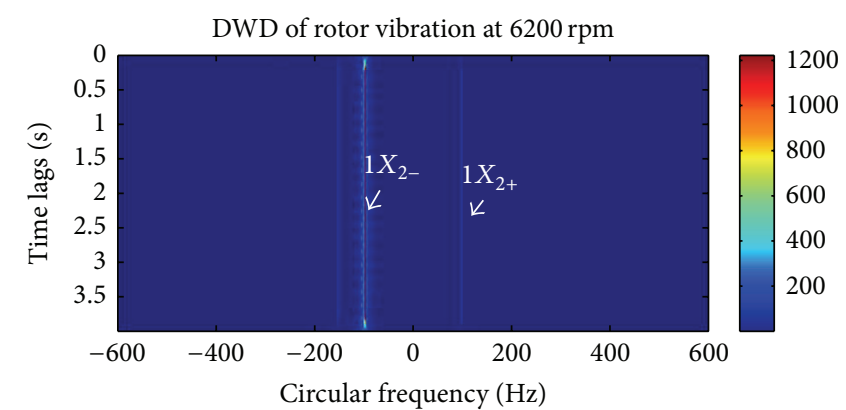

(b)

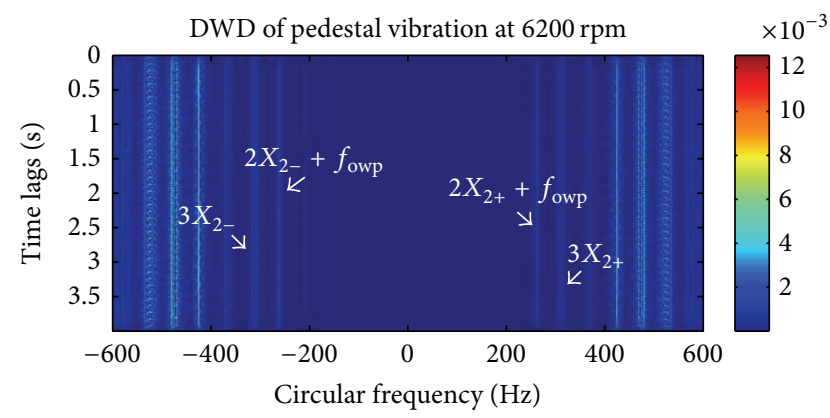

(d)

FIGURE 10: Directional Wigner distribution of the lateral vibration of the shaft and pedestal.

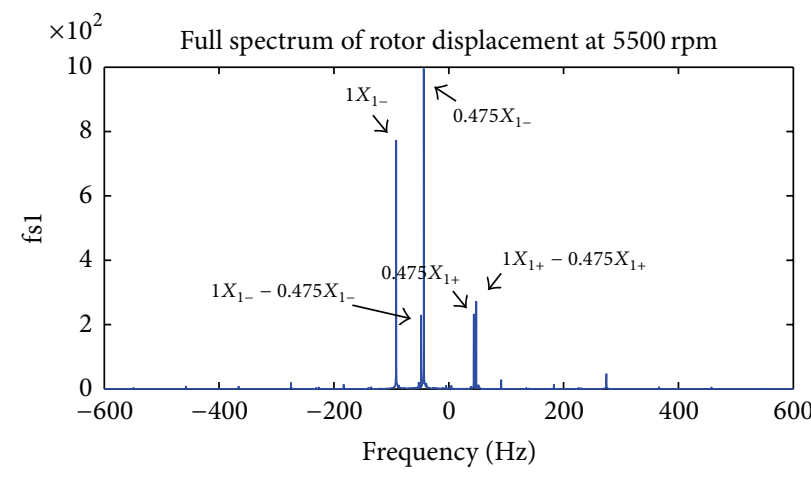

(a)

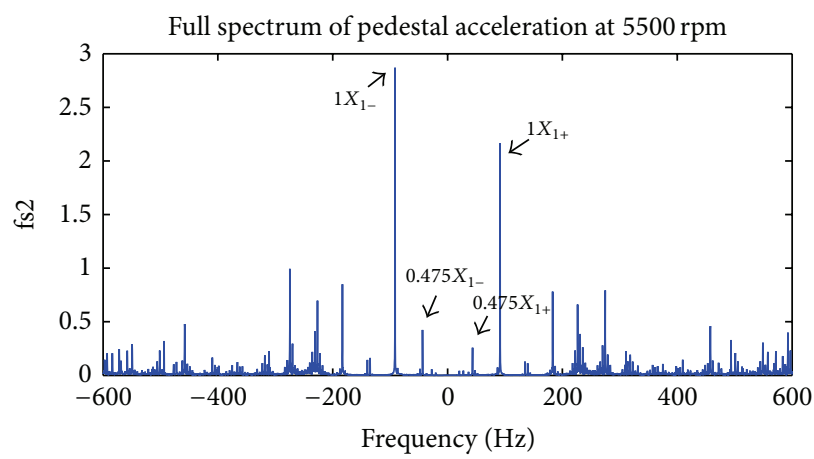

(c)

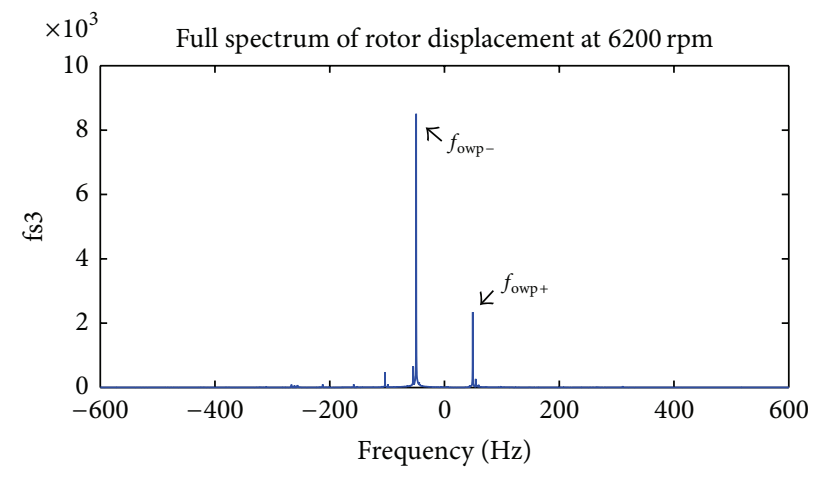

(b)

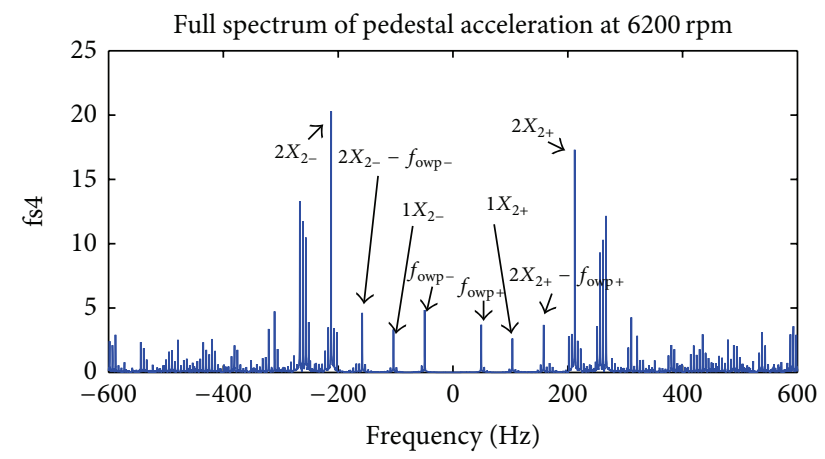

(d)

FIGURE 11: Full spectrum of the lateral vibration of the shaft and pedestal. 


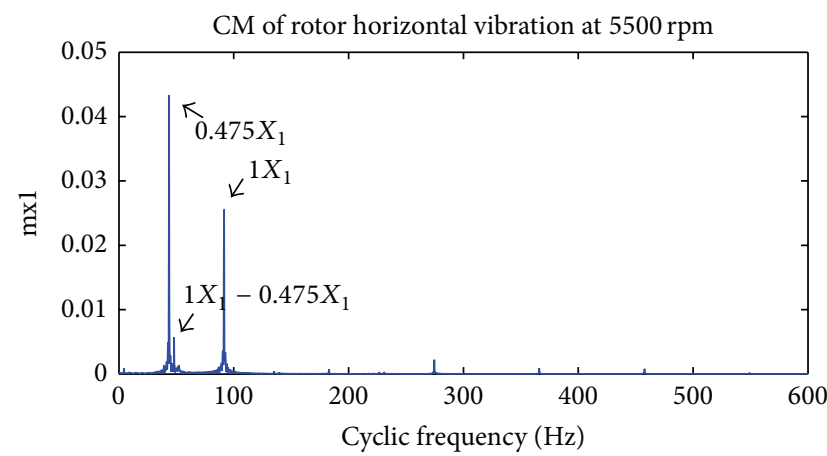

(a)

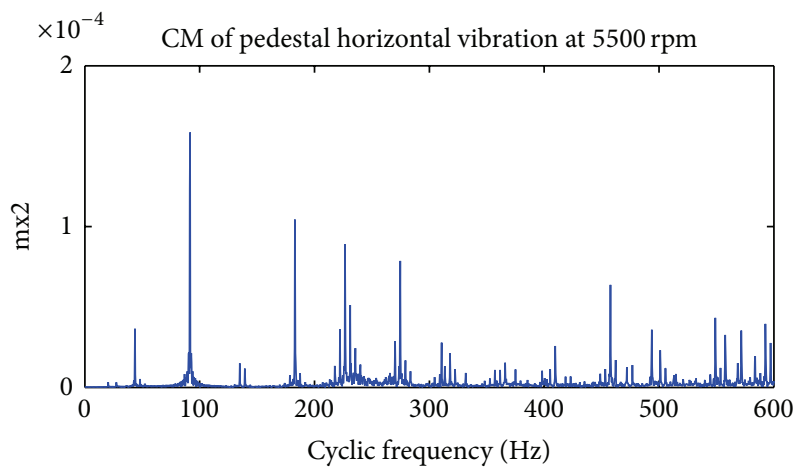

(c)

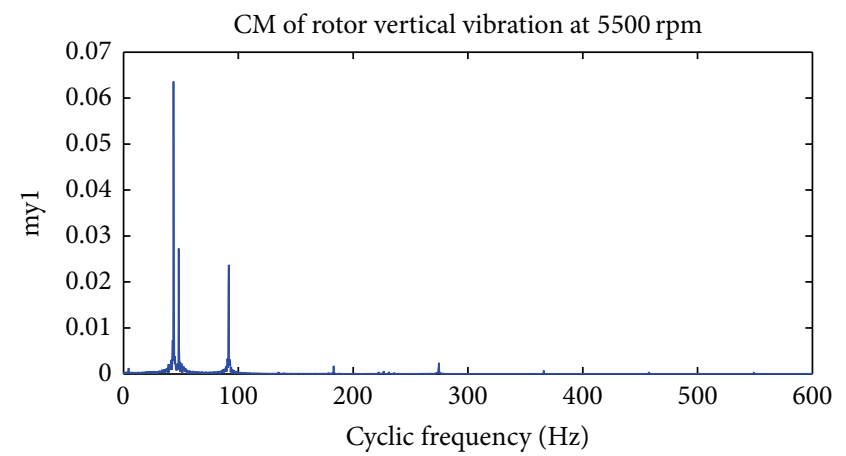

(b)

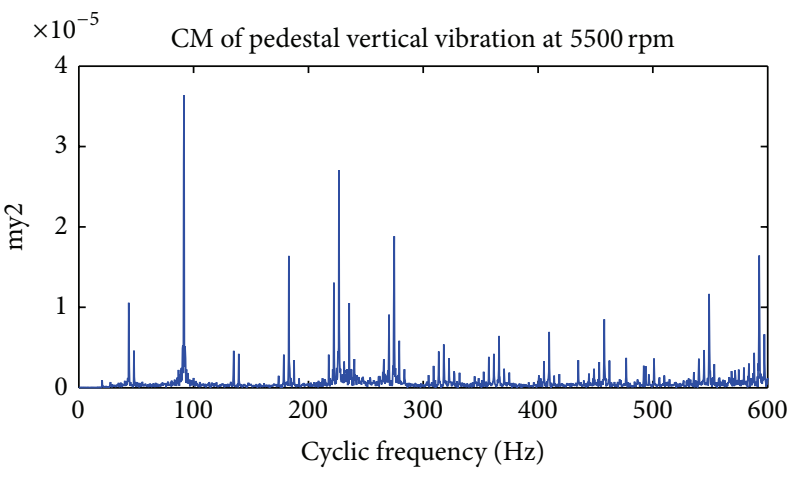

(d)

Figure 12: The cyclic mean of the lateral vibration of the shaft and pedestal at $5500 \mathrm{rpm}$.

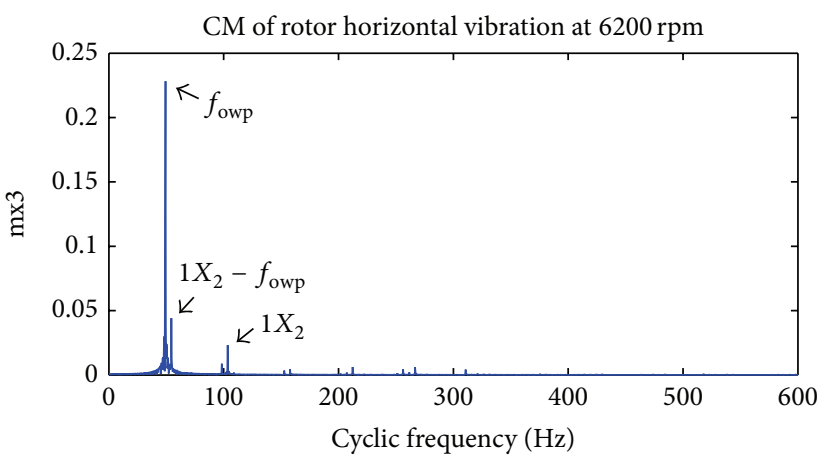

(a)

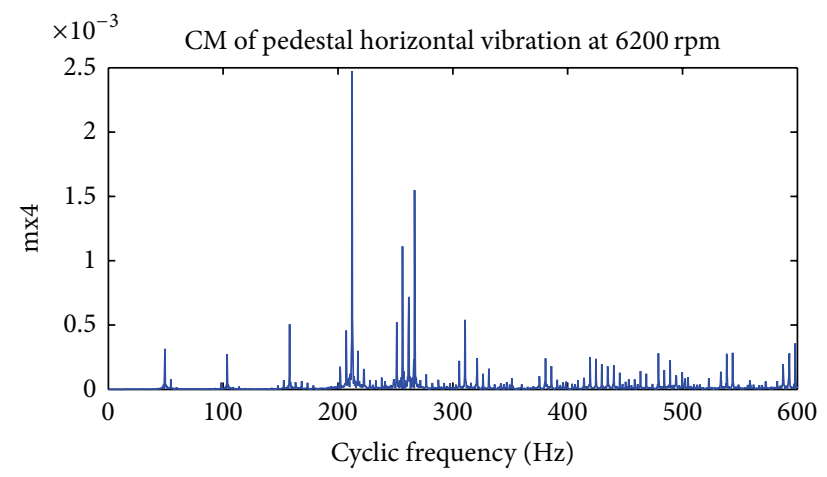

(c)

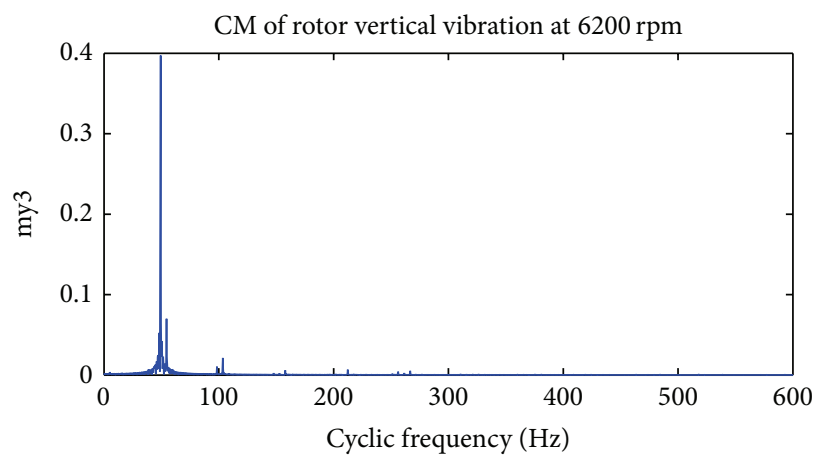

(b)

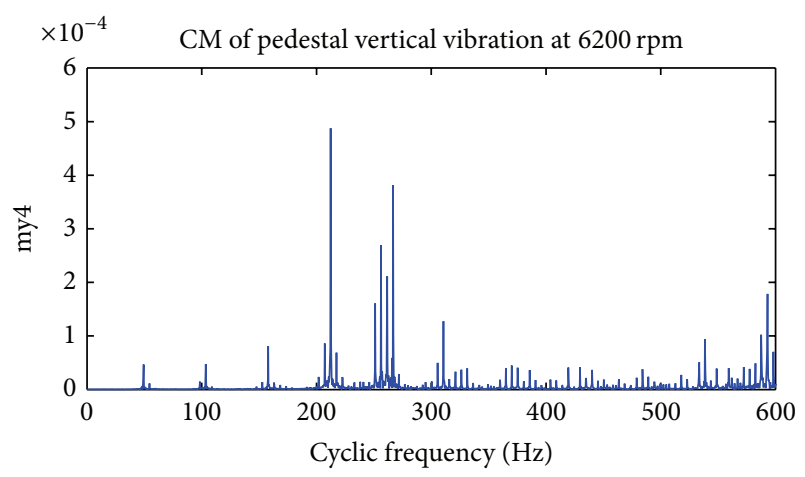

(d)

FIGURE 13: The cyclic mean of the lateral vibration of the shaft and pedestal at $6200 \mathrm{rpm}$. 


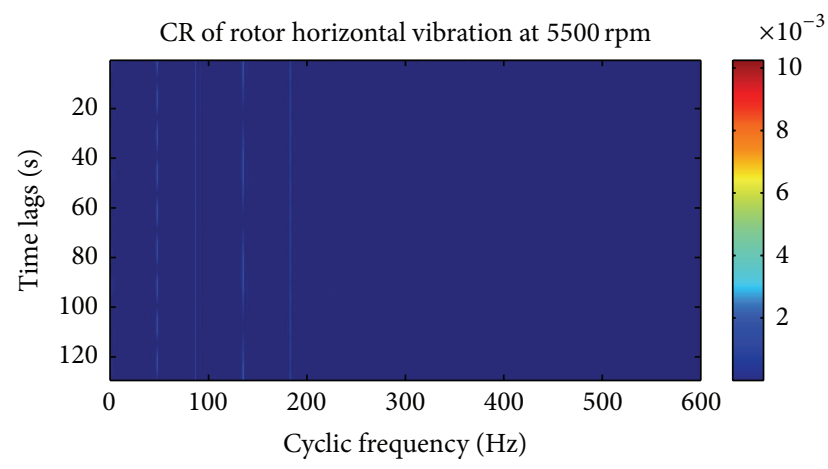

(a)

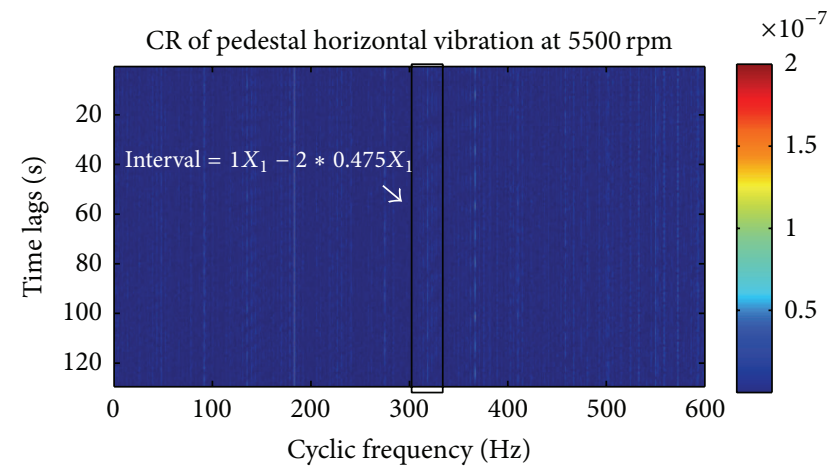

(c)

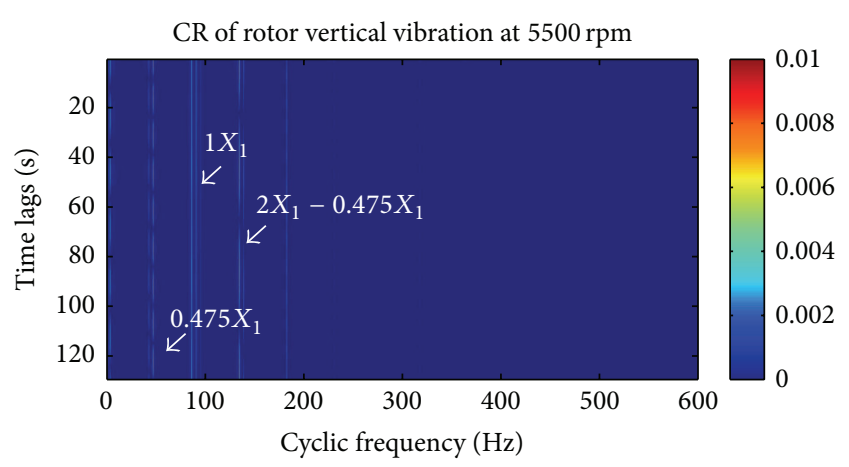

(b)

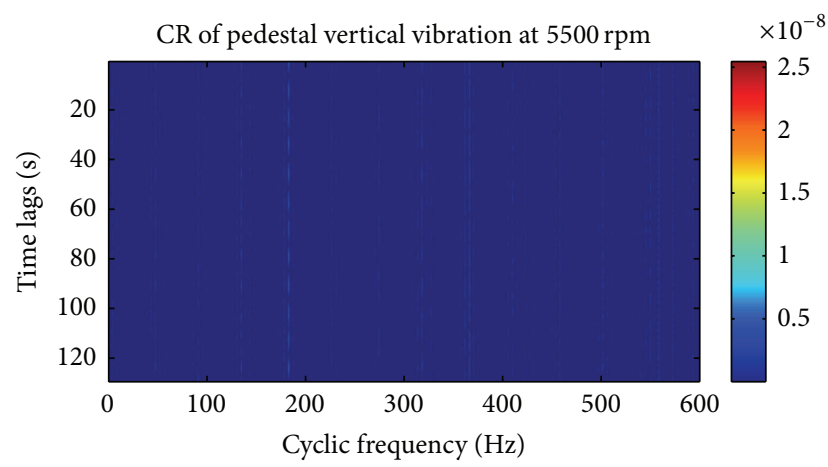

(d)

FIGURE 14: The cyclic autocorrelation of the lateral vibration of the shaft and pedestal at $5500 \mathrm{rpm}$.

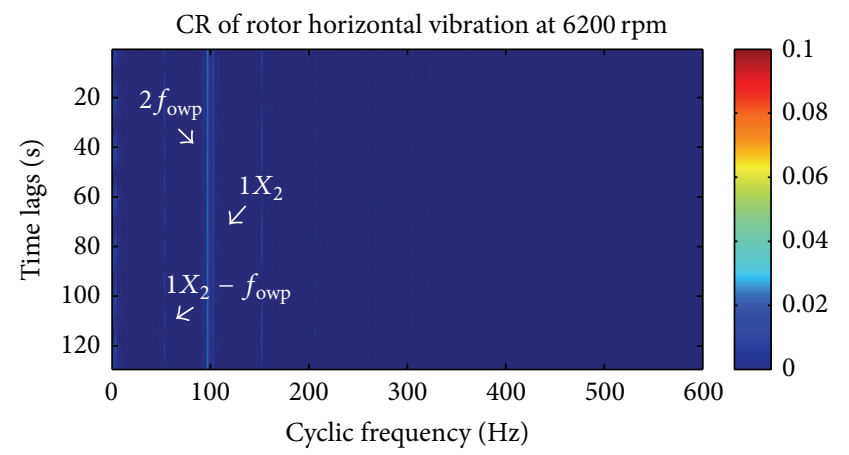

(a)

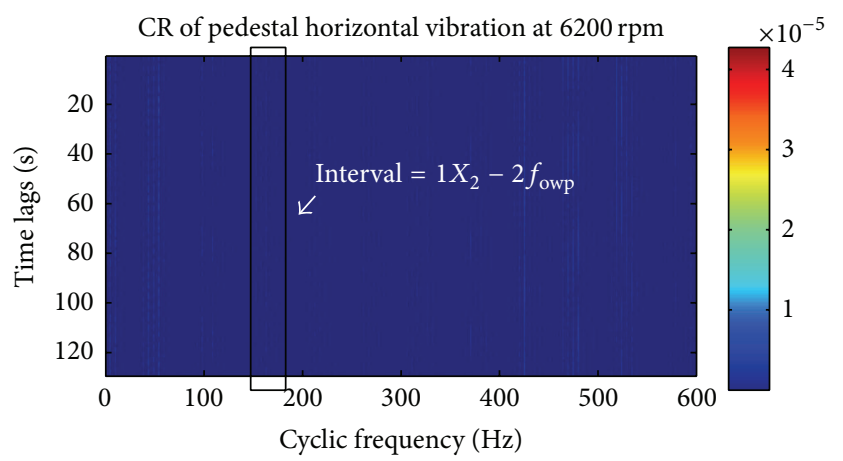

(c)

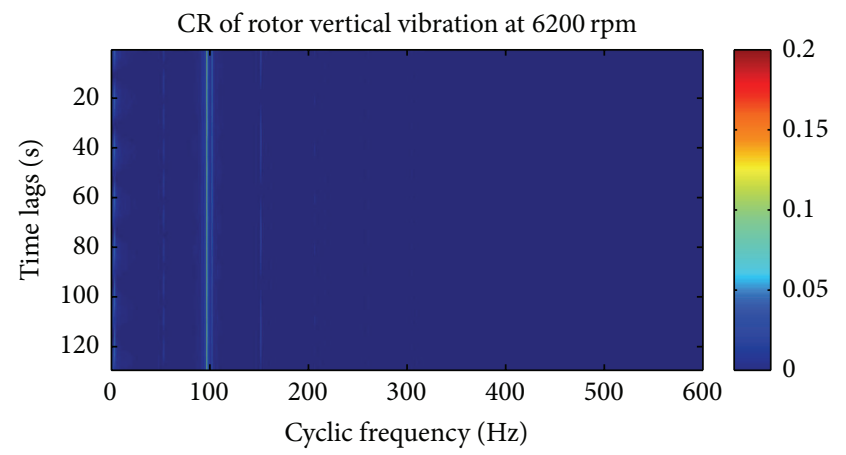

(b)

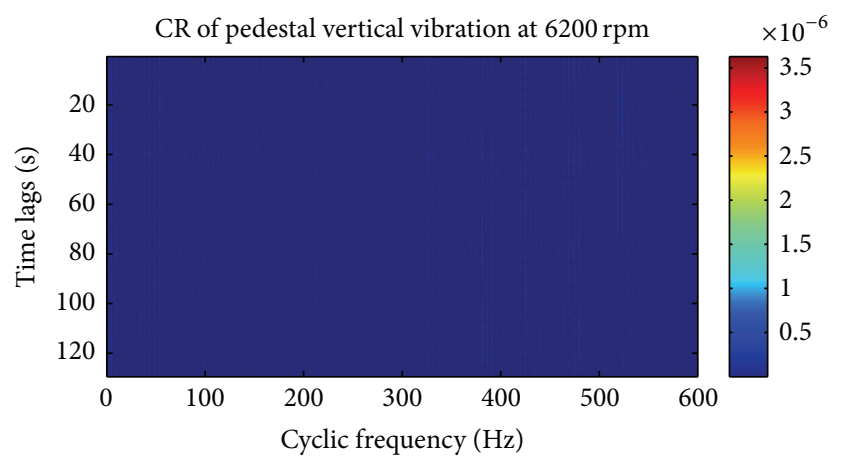

(d)

FIGURE 15: The cyclic autocorrelation of the lateral vibration of the shaft and pedestal at $6200 \mathrm{rpm}$. 
where $\operatorname{DCM}_{f}(\alpha, t)$ and $\operatorname{DCM}_{b}(\alpha, t)$ are, respectively, the forward and backward terms. DCM is able to identify the periodic components induced by the mean behavior of the signal.

The autodirectional cyclic autocorrelation (DCR) is defined in (11) according to (2) and (7):

$$
\begin{aligned}
& \operatorname{DCR}_{p p^{*}}(\alpha, \tau) \\
& = \begin{cases}\operatorname{DCR}_{p^{f} p^{f^{*}}}(\alpha, \tau) \\
\operatorname{DCR}_{p^{b} p^{b^{*}}}(\alpha, \tau)\end{cases} \\
& = \begin{cases}\sum_{\alpha \in \widetilde{A}} E\left[p^{f}\left(t-\frac{\tau}{2}\right) p^{f^{*}}\left(t+\frac{\tau}{2}\right) \cdot e^{-2 \pi \alpha t}\right], & \text { for } \alpha>0 \\
\sum_{\alpha \in \widetilde{A}} E\left[p^{b}\left(t-\frac{\tau}{2}\right) p^{b^{*}}\left(t+\frac{\tau}{2}\right) \cdot e^{-2 \pi \alpha t}\right], & \text { for } \alpha<0,\end{cases}
\end{aligned}
$$

where $\operatorname{DCR}_{p^{f} p^{f^{*}}}(\alpha, \tau)$ and $\operatorname{DCR}_{p^{b} p^{b^{*}}}(\alpha, \tau)$ are, respectively, the forward and backward terms.

The cross-directional cyclic autocorrelation is given in (12) according to (2) and (8):

$$
\begin{aligned}
& \operatorname{DCR}_{p^{b^{*}} p^{f}}(\alpha, \tau) \\
& =\sum_{\alpha \in \widetilde{A}} E\left[p^{b}\left(t-\frac{\tau}{2}\right) p^{f}\left(t+\frac{\tau}{2}\right) \cdot e^{-2 \pi \alpha t}\right], \quad \forall \alpha .
\end{aligned}
$$

Both the auto- and the cross-directional cyclic autocorrelation coefficients can indicate the energy of the signal.

Subsequently, taking the Fourier transform of the autodirectional cyclic autocorrelation with respect to the lag $\tau$ produces the auto directional spectral correlation density function (DSCD), given by (13) according to (3) and (7):

$$
\begin{aligned}
& \operatorname{DSCD}_{p p^{*}}(\alpha, f) \\
& = \begin{cases}\operatorname{DSCD}_{p^{f} p^{f^{*}}}(\alpha, f) \\
\operatorname{DSCD}_{p^{b} p^{b^{*}}}(\alpha, f)\end{cases} \\
& = \begin{cases}\int_{-\infty}^{\infty} \operatorname{DCR}_{p^{f} p^{f^{*}}}(\alpha, \tau) \cdot e^{-2 \pi f \tau} d \tau, & \text { for } \alpha>0 \\
\int_{-\infty}^{\infty} \operatorname{DCR}_{p^{b} p^{b^{*}}}(\alpha, \tau) \cdot e^{-2 \pi f \tau} d \tau, & \text { for } \alpha<0 .\end{cases}
\end{aligned}
$$

In the particular case when the cyclic frequency $\alpha=0$, (13) becomes the power spectrum of the signal $p(t)$.

The cross-directional spectral correlation density function is given by (14) according to (3) and (8):

$$
\operatorname{DSCD}_{p^{b^{*}} p^{f}}(\alpha, f)=\int_{-\infty}^{\infty} \operatorname{DCR}_{p^{b^{*}} p^{f}}(\alpha, \tau) \cdot e^{-2 \pi f \tau} d \tau, \quad \forall \alpha .
$$

Both the auto- and the cross-directional spectral correlation density functions actually describe the density of the correlation of two spectral components spaced apart by $\alpha$ around the central frequency $f$.
TABLE 1: System operating states at different rotating speeds.

\begin{tabular}{lcl}
\hline Experiment & Operating speed & $\begin{array}{c}\text { Observed state of } \\
\text { operation }\end{array}$ \\
\hline 1 & $5500 \mathrm{rpm}$ & Oil whirl \\
2 & $6200 \mathrm{rpm}$ & Oil whip \\
\hline
\end{tabular}

As the frequency $f$ in the conventional Wigner-Ville spectrum does not represent the periodicity of the vibration response as the cyclic frequency $\alpha$ does, the negative component of the frequency $f$ will be of no meaning. Therefore, by transforming the cyclic frequency variable $\alpha$ back to the time domain, no variable with physical meaning can be obtained, which means that the directional Wigner-Ville spectrum does not exist.

It is noted that all the significant symbols in the equations are listed in the Nomenclature section.

\section{Experiment}

3.1. Experimental Setup and Data Description. In this work, the experiment is conducted to investigate the dynamics of the asymmetric journal bearing supported rotor system on a test rig illustrated in Figure 1. The experiment setup consists of a rigid cylindrical shaft supported by two cylindrical journal bearings, with the supporting journal bearing on the right end near the driving motor and an oil film journal bearing at the left end for simulating oil film instability faults. Two discs are mounted on the shaft, with one at the midplane between the two bearings and the other near the left oil film bearing. Two pairs of accelerometers are used for measuring pedestal translational vibrations, with one pair mounted on the outboard of each of the two bearing pedestals. In addition, two proximity sensors are mounted just to the right-hand side of the center disc, to record the lateral vibrations of the rotor at that position.

In order to simulate the oil whirl and oil whip phenomenon, the rotor rotating in fluid lubricated cylindrical journal bearings is lightly loaded with an unbalanced mass. When the shaft rotates with a slow rotating speed, the stable synchronous vibration with low amplitude is observed. With the increasing rotation speed, the system reaches the first resonance with significant vibration amplitude at the first natural frequency. Then the vibration returns to normal after the resonance. At higher speed, the oil whirl appears along with higher vibration. In addition, the characteristic frequency of oil whirl (around half of the rotating frequency) can be observed. When the rotating speed approaches the double resonance speed, the oil whirl pattern becomes the oil whip with the characteristic frequency remaining to the first natural frequency of the system. Furthermore, the amplitude of the rotor under oil whip becomes higher than that of oil whirl. The vibration signals of journal bearing supported system during startup procedure are listed in Figure 2. Results obtained under different rotational speeds with various artificially simulated defects are listed in Table 1. 


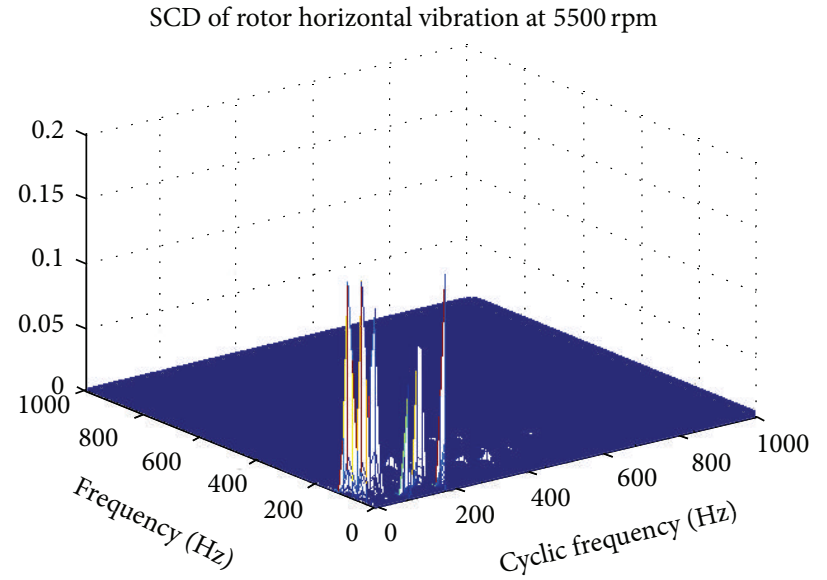

(a)

SCD of pedestal horizontal vibration at $5500 \mathrm{rpm}$

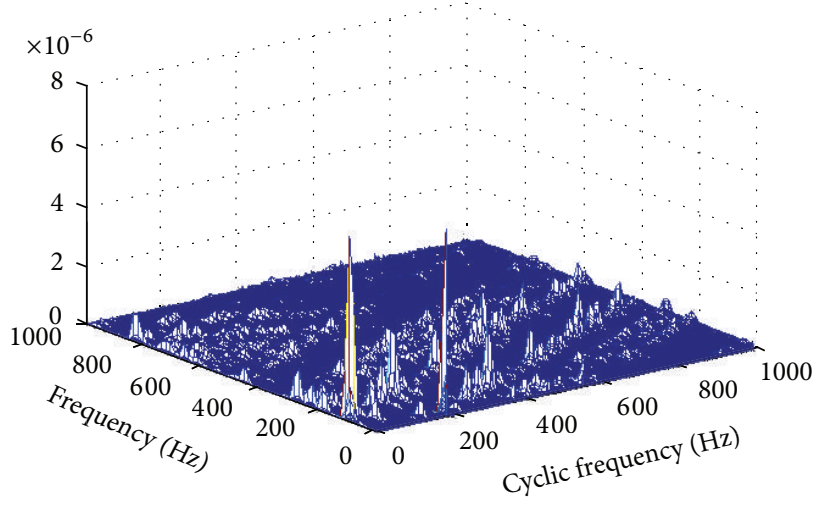

(c)

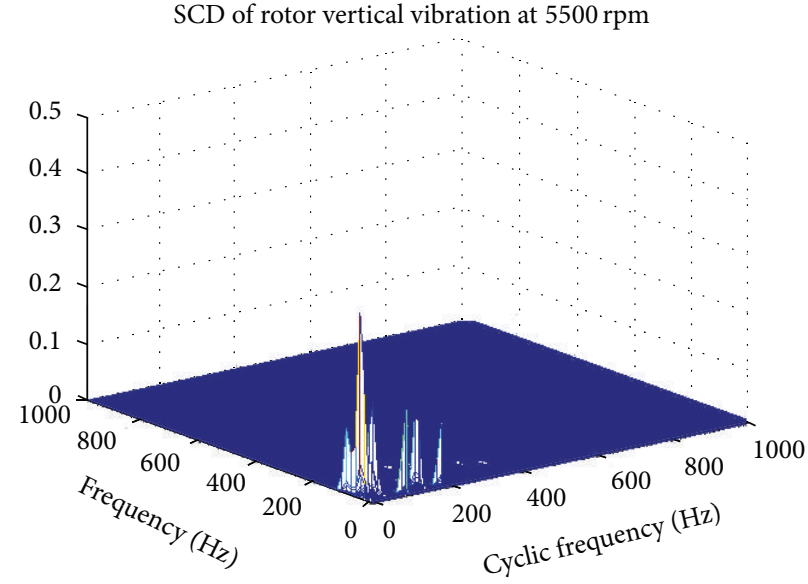

(b)

SCD of pedestal vertical vibration at $5500 \mathrm{rpm}$

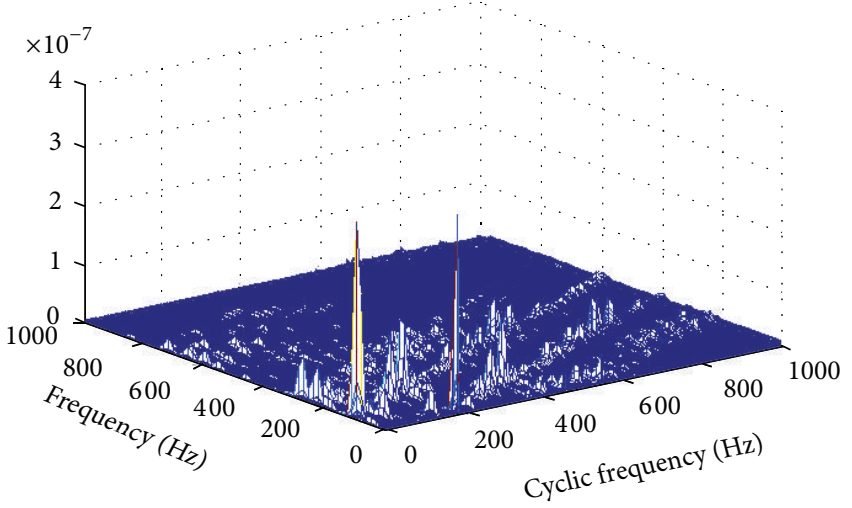

(d)

FIGURE 16: The spectral correlation density of the lateral vibration of the shaft and pedestal at $5500 \mathrm{rpm}$.

3.2. Vibration Signal Analysis. The vibration response of the shaft and the pedestal when the system operated at a rotational speed of $5500 \mathrm{rpm}$ and $6200 \mathrm{rpm}$ was investigated using directional cyclostationarity, directional time frequency distribution, and conventional cyclostationarity, respectively. In order to facilitate calculation, the first 8192 samples of the original signal and their power spectrum derived with sampling frequency $f_{s}=2048 \mathrm{~Hz}$ were selected and shown in Figures 3, 4, 5, and 6.

\subsubsection{Directional Cyclostationarity of the Complex-Valued} Signal. Firstly, in this work, the directional cyclostationary parameters of the complex-valued vibration signal of the rotor-bearing system were derived to help extract the hidden periodicity of nonstationary signature, as well as investigating the instantaneous planar motion.

In Figure 7, the positive (negative) horizontal axes represent the first order cyclostationarity, namely, the cyclic mean, of the forward (backward) vectors of the rotor lateral vibration. In Figure 7(a) derived from the rotor vibration at $5500 \mathrm{rpm}$, the oil whirl frequency $0.475 X_{1}$ and the rotating frequency $1 X_{1}$ and its value modulated by the oil whirl frequency $1 X_{1}-0.475 X_{1}$ can be clearly identified. Figure $7(\mathrm{~b})$ characterizing the rotor vibration at $6200 \mathrm{rpm}$ only displays the oil whip frequency $f_{\text {owp }}=49.34 \mathrm{~Hz}$ but misses the rotating frequency. However, Figures 7 (c) and $7(\mathrm{~d})$ derived from pedestal vibration provide more abundant frequency information. The harmonics $\pm 2 X_{2}$ of rotating frequency dominates the horizontal axes in Figure $7(\mathrm{~d})$ of pedestal vibration at $6200 \mathrm{rpm}$. Furthermore, the harmonics of rotating frequency $X_{2}$ modulated by the oil whip frequency is also clear. In addition, the developed directional cyclic mean can help determine the instantaneous procession orientation of the rotor centerline. With $R_{1 X_{1^{-}}}>R_{1 X_{1}+}$ or $R_{1 X_{2^{-}}}>R_{1 X_{2^{+}}}$, the direction will be backward or opposite to the direction of rotor rotation.

In Figure 8, the positive (negative) horizontal axes give the second order cyclostationarity, namely, the cyclic autocorrelation, of the forward (backward) vectors of the shaft and pedestal lateral vibration. It can be seen that the rotating frequency modulated by oil whirl frequency (oil whip frequency) appears periodically in the DCR plot derived from rotor or pedestal vibration when system operates at $5500 \mathrm{rpm}(6200 \mathrm{rpm})$, while the harmonics of the single rotating frequency keeps all the same. In addition, the 


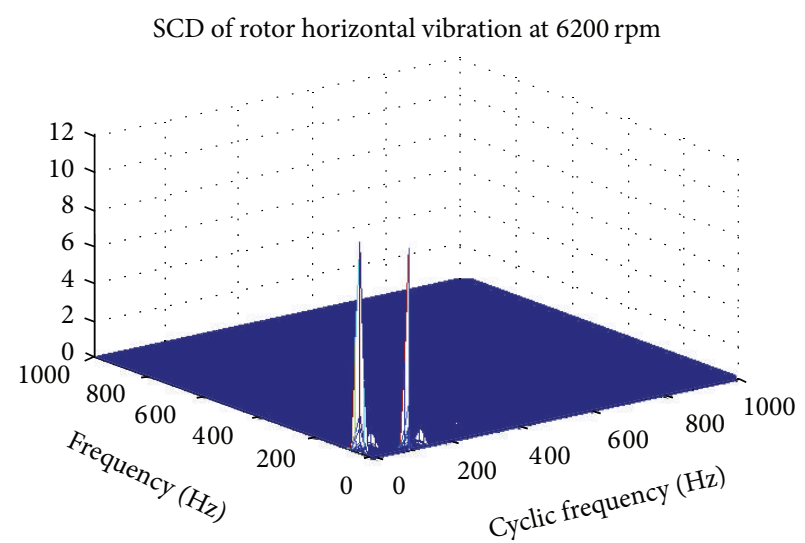

(a)

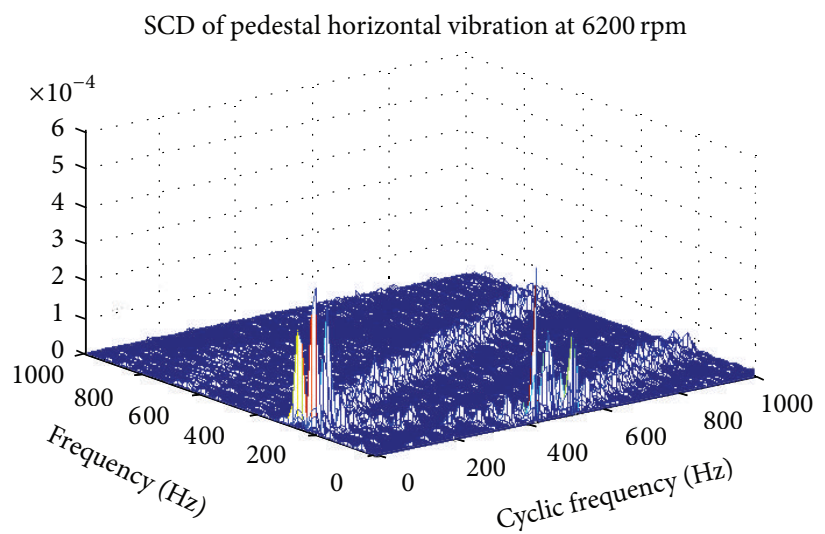

(c)

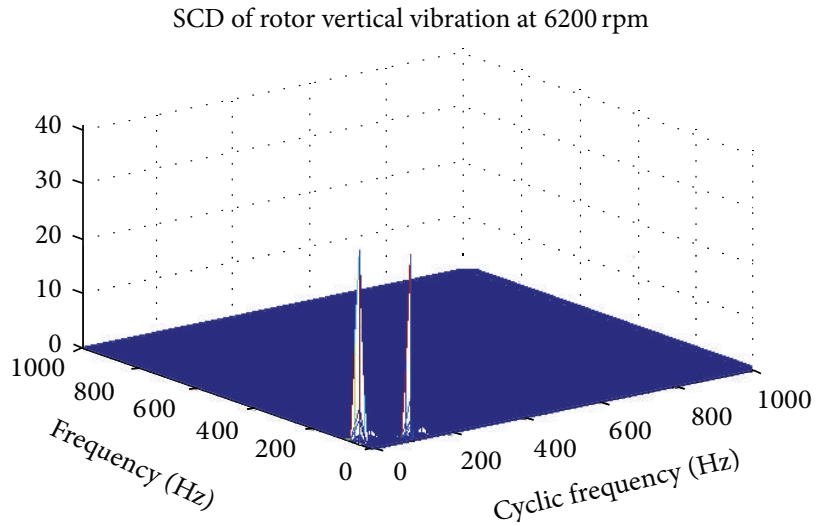

(b)

SCD of pedestal vertical vibration at $6200 \mathrm{rpm}$

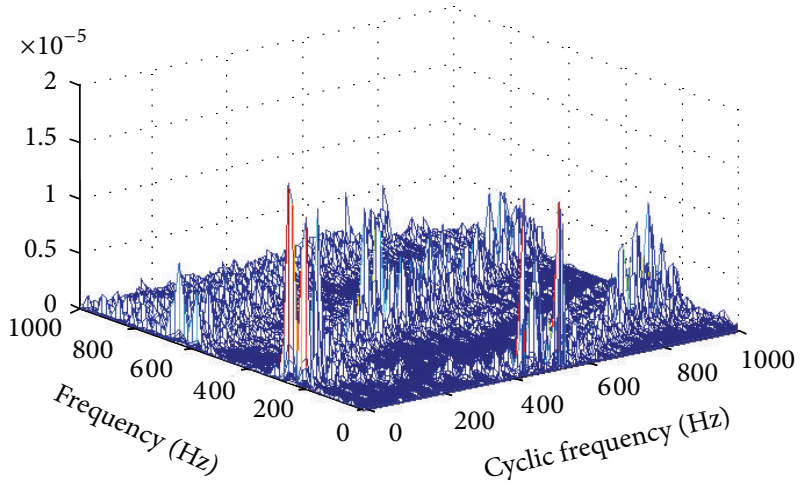

(d)

FIgURE 17: The spectral correlation density of the lateral vibration of the shaft and pedestal at $6200 \mathrm{rpm}$.

instantaneous procession orientation of the rotor centerline can be determined by comparing the color of the positive and negative value which represents the radii of the orbit.

As defined in [10], the SCD plots comprised of countable nonzero points show the first order cyclostationarity, while the SCD plots comprised of parallel lines exhibit the second order cyclostationarity. In Figure 9, the SCD plots of the pedestal vibration signal clearly represent that the density of the correlation continuously changes along several straight lines in the frequency cyclic frequency plane. Therefore, it can be inferred that the case of vibrations induced by oil whirl or oil whip is more suitable to be interpreted as the second order cyclostationarity. In addition, the DSCD plots of the pedestal vibration are more suitable to interpret the case induced by oil whirl or oil whip than that of the rotor vibration. Besides, the DSCD plots also can help determine the procession direction by comparing the amplitudes of the density of the correlation at positive and negative cyclic rotating frequency.

3.2.2. Directional Wigner Distribution. Subsequently, directional time-frequency distributions of the complex-valued signal derived from the lateral vibration of the journal bearing supported rotor system are given in Figure 10 for comparison.
As seen in Figure 10(a) derived from rotor displacement when system operates at $5500 \mathrm{rpm}$, the orbit radius when $\omega=$ $1 X_{1}$ is much larger than that when $\omega=-1 X_{1}$. With $R_{1 X_{1}-}>$ $R_{1 X_{1}+}$, the instantaneous precession orientation of the rotor centerline will be backward. As the oil whip frequency component dominates the whole frequency band in Figure 10(b) of the rotor vibration, the rotating frequency component cannot be clearly seen. However, in Figures 10(c) and 10(d) derived from pedestal vibration, the rotating frequency $\pm X_{2}$, the oil whip frequency $\pm f_{\text {owp }}$, and the modulating frequency harmonics comprised of $X_{2}$ and $f_{\text {owp }}$ can be clearly seen. In addition, it also can be seen that the rotating frequency modulated by oil whirl or oil whip appears periodically versus time while the harmonics of single rotating frequency exists all the time as the time changes.

By further comparing the directional Wigner distribution defined by (7) to (8) with the directional cyclic autocorrelation defined by (11) to (12), it is interesting to find that actually the directional Wigner distribution can be interpreted as the second order directional cyclostationarity (directional cyclic autocorrelation) characterizing the random behavior of the system. Furthermore, it is found that the full spectrum can be interpreted as the first order directional cyclostationarity 

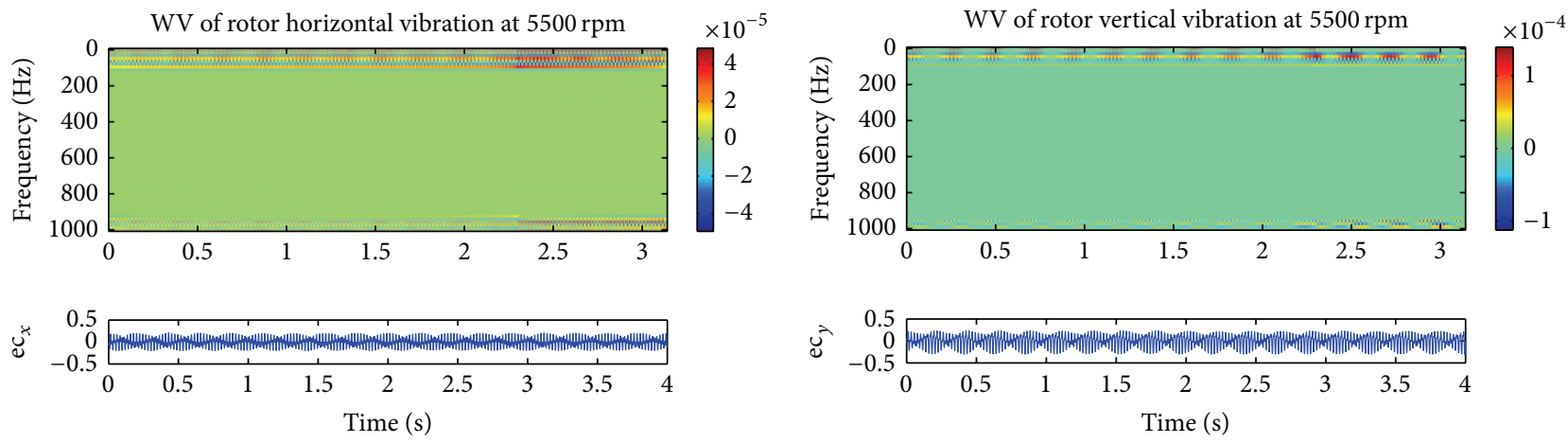

(a)

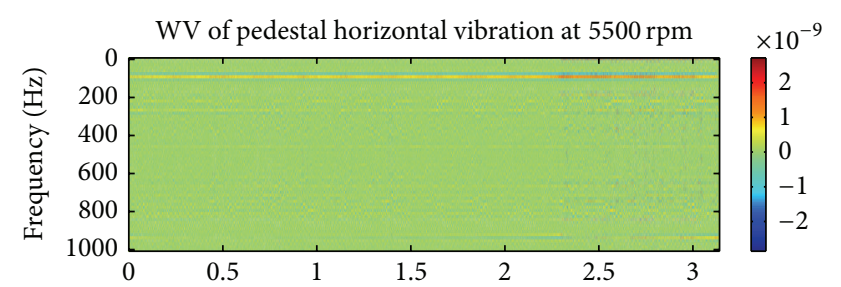

(b)

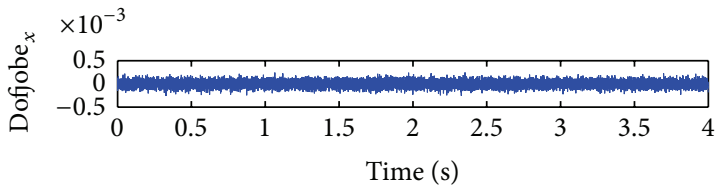

(c)
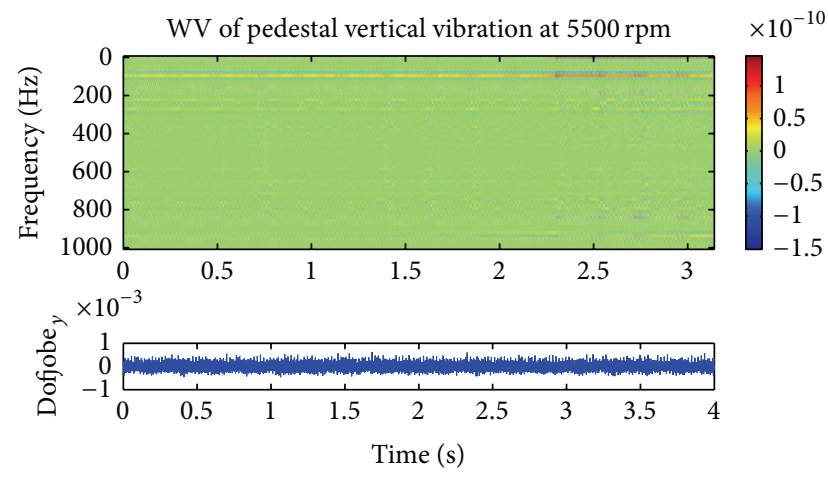

(d)

Figure 18: The Wigner-Ville spectrum of the lateral vibration of the shaft and pedestal at $5500 \mathrm{rpm}$.

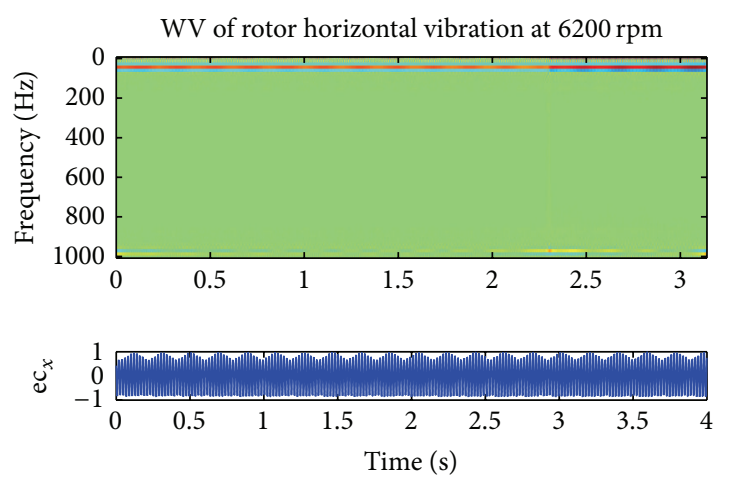

(a)
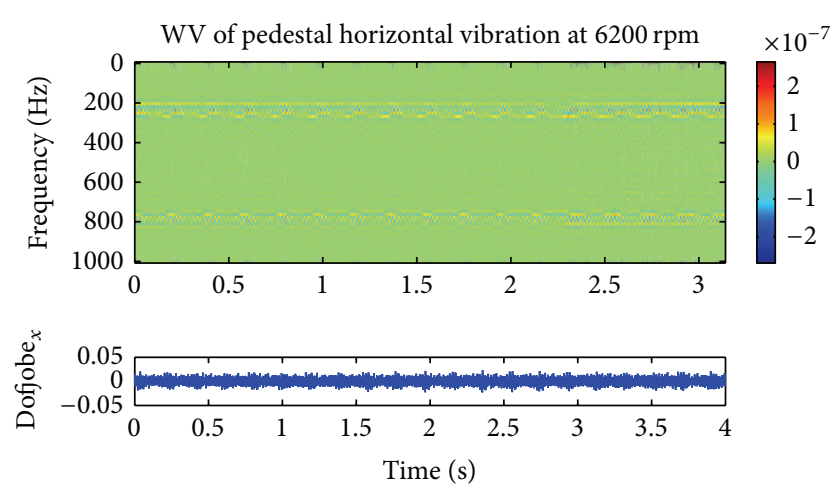

(c)
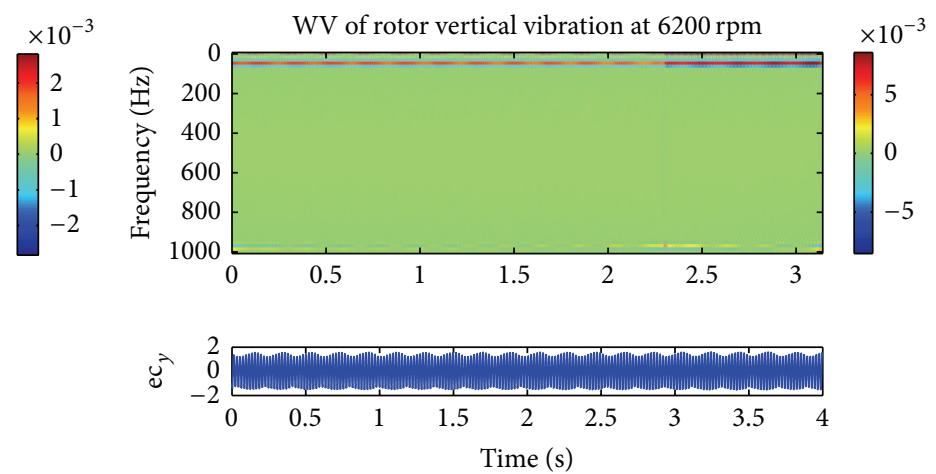

(b)
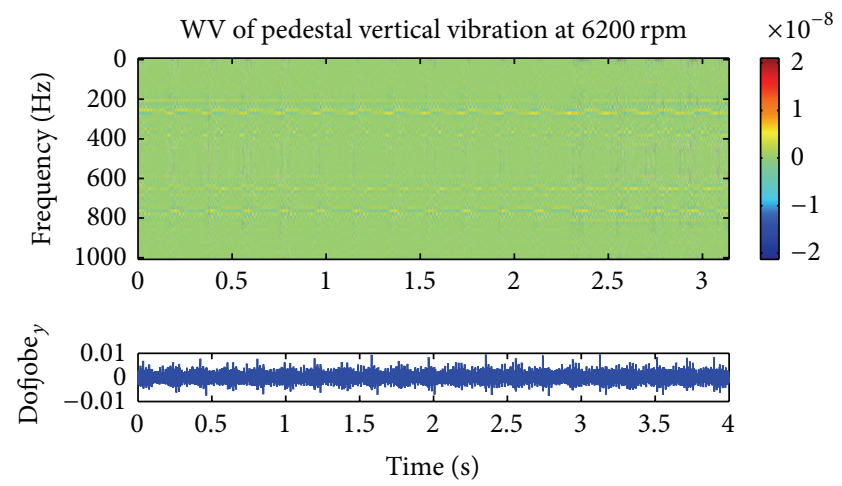

(d)

FIgURE 19: The Wigner-Ville spectrum of the lateral vibration of the shaft and pedestal at $6200 \mathrm{rpm}$. 
(directional cyclic mean) by comparing (9) and (10), as shown in Figures 11 and 7.

3.2.3. Cyclostationarity of the Real-Valued Signal. For completeness, the statistical parameters characterizing cyclostationarity of real-valued signal of the journal bearing supported system are also illustrated from Figures 12, 13, 14, $15,16,17,18$, and 19. Similar conclusions to the directional cyclostationarity plots can be found except for information on the procession directivity of the planar motion.

Compared with conventional Wigner-Ville distribution, the Wigner-Ville spectrum reduces the influence of the interference terms on exhibiting how the signal energy flows in the time and frequency domain [10]. In Figure 18, only the WV Figures 18(a) and 18(b) derived from the rotor vibration are able to clearly describe the periodicity induced by oil whirl fault. In Figure 19, both the WV plots of the rotor vibration and the pedestal vibration obviously show the periodic phenomenon responding to oil whip fault.

\section{Discussions and Conclusions}

In the work presented here, the characteristics of the vibration signals obtained both from the rotor and the pedestal of a rotor system supported by journal bearing were investigated. The experiments were conducted on a test bench to simulate the operation status of the rotor system suffering from oil whirl and oil whip.

The directional cyclostationary parameters, such as directional cyclic mean, directional cyclic autocorrelation, and directional spectral correlation density, were defined and utilized to analyze the complex-valued lateral vibration signal of the rotor system. The proposed method is able to identify the oil whirl or oil whip fault. In addition, the information on the procession directivity of the planar motion can also be obtained. Furthermore, it is found that actually the full spectrum can be interpreted as the first order directional cyclostationarity and the directional Wigner distribution as the second order directional cyclostationarity. Therefore the developed directional cyclostationarity integrates both the advantages of conventional cyclostationarity and the directional Wigner distribution.

\section{Nomenclature}

$\operatorname{CM}(\alpha): \quad$ Cyclic mean at $\alpha$

$\operatorname{CR}(\alpha, \tau): \quad$ Cyclic autocorrelation at $(\alpha, \tau)$

$\operatorname{SCD}(\alpha, f)$ : The spectral correlation density at $(\alpha, f)$

$\mathrm{WV}(t, f)$ : The Wigner-Ville spectrum at $(t, f)$

$\alpha: \quad$ Cyclic frequency

$\tau: \quad$ Time lag

$p(t): \quad$ Complex-valued vibration signal

$x(t)$ : $\quad$ Real-valued vibration signal in $x$ direction

$y(t): \quad$ Real-valued vibration signal in $y$ direction

$p^{f}(t): \quad$ Forward complex-valued vibration signal $p^{b}(t)$

$W_{p}^{d}(t, \omega):$

$W_{p^{*}, p}^{d}(t, \omega)$ :

$\operatorname{FS}(t, \omega)$ :

$\operatorname{DCM}(\alpha)$ :

$\operatorname{DCR}_{p p^{*}}(\alpha, \tau): \quad$ Autodirectional cyclic autocorrelation at

$\operatorname{DCR}_{p^{b^{*} p^{f}}}(\alpha, \tau): \quad$ Cross-directional cyclic autocorrelation at $(\alpha, \tau)$

$\operatorname{DSCD}_{p p^{*}}(\alpha, f)$ : Autodirectional spectral correlation density function at $(\alpha, f)$

$\operatorname{DSCD}_{p^{b^{*}} p^{f}}(\alpha, f)$ : Cross-directional spectral correlation density function at $(\alpha, f)$.

\section{Conflict of Interests}

The authors declare that there is no conflict of interests regarding the publication of this paper.

\section{Acknowledgment}

This work is supported by the Fundamental Research Funds for the Central Universities (no. CDJZR12115501).

\section{References}

[1] A. Muszynska, "Whirl and whip-Rotor/bearing stability problems," Journal of Sound and Vibration, vol. 110, no. 3, pp. 443462, 1986.

[2] A. Muszynska, "Stability of whirl and whip in rotor/bearing systems," Journal of Sound and Vibration, vol. 127, no. 1, pp. 4964, 1988.

[3] A. Moosavian, H. Ahmadi, A. Tabatabaeefar, and M. Khazaee, "Comparison of two classifiers; K-nearest neighbor and artificial neural network, for fault diagnosis on a main engine journal-bearing," Shock and Vibration, vol. 20, no. 2, pp. $263-$ 272, 2013.

[4] J. Ying, Y. Jiao, and Z. Chen, "Nonlinear dynamics analysis of tilting pad journal bearing-rotor system," Shock and Vibration, vol. 18, no. 1-2, pp. 45-52, 2011.

[5] T. W. Dimond, P. N. Sheth, P. E. Allaire, and M. He, "Identification methods and test results for tilting pad and fixed geometry journal bearing dynamic coefficients-a review," Shock and Vibration, vol. 16, no. 1, pp. 13-43, 2009.

[6] A. Zanarini and A. Cavallini, "Experiencing rotor and fluid film bearing dynamics," in Proceedings of the Quinta Giornata di Studio Ettore Funaioli, pp. 23-38, Bologna, Italy, 2011.

[7] W. A. Gardner, A. Napolitano, and L. Paura, "Cyclostationarity: half a century of research," Signal Processing, vol. 86, no. 4, pp. 639-697, 2006.

[8] E. Serpedin, F. Panduru, I. Sari, and G. B. Giannakis, "Bibliography on cyclostationarity," Signal Processing, vol. 85, no. 12, pp. 2233-2303, 2005.

[9] A. C. Mccormick and A. K. Nandi, "Cyclostationarity in rotating machine vibrations," Mechanical Systems and Signal Processing, vol. 12, no. 2, pp. 225-242, 1998. 
[10] J. Antoni, "Cyclostationarity by examples," Mechanical Systems and Signal Processing, vol. 23, no. 4, pp. 987-1036, 2009.

[11] D. Southwick, "Using full spectrum plots," Orbit, vol. 14, pp. 1921, 1993.

[12] D. Southwick, "Using full spectrum plots-part 2," Orbit, vol. 15, pp. 11-15, 1994.

[13] C.-W. Lee, Y.-S. Han, and J.-P. Park, "Use of directional spectra for detection of engine cylinder power fault," Shock and Vibration, vol. 4, no. 5-6, pp. 391-401, 1997.

[14] C. W. Lee and Y.-S. Han, "The directional Wigner distribution and its applications," Journal of Sound and Vibration, vol. 216, no. 4, pp. 585-600, 1998.

[15] Y.-S. Han and C.-W. Lee, "Directional Wigner distribution for order analysis in rotating/reciprocating machines," Mechanical Systems and Signal Processing, vol. 13, no. 5, pp. 723-738, 1999. 

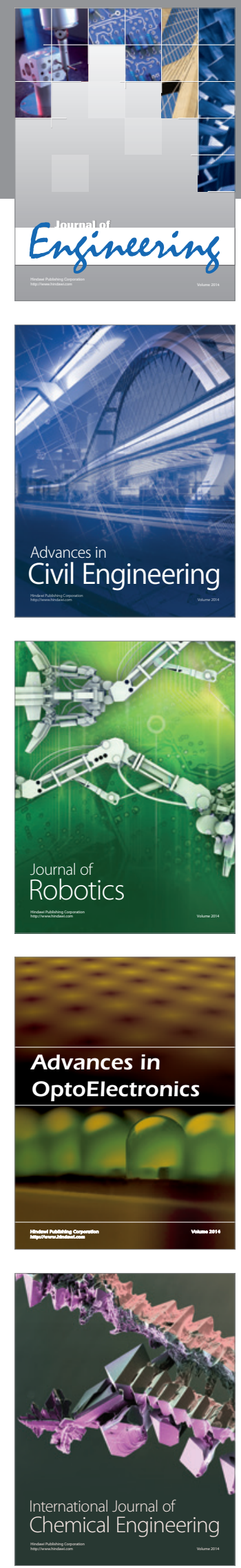

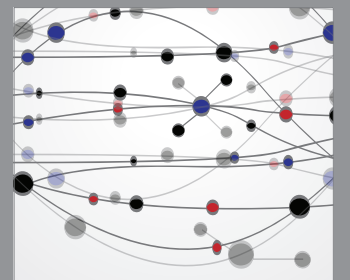

The Scientific World Journal
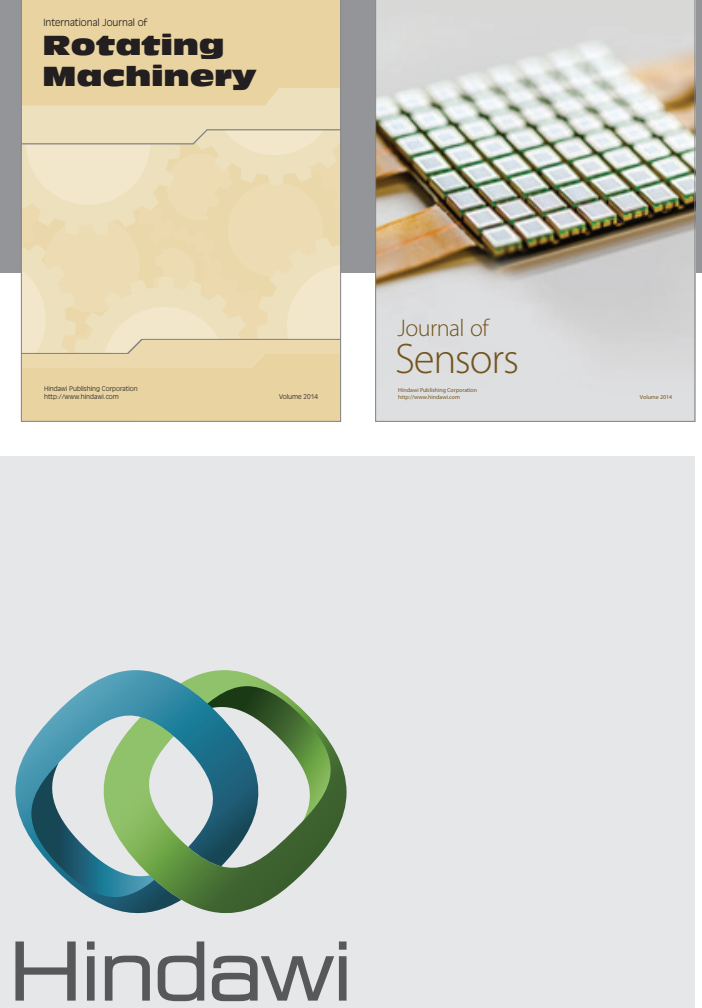

Submit your manuscripts at http://www.hindawi.com
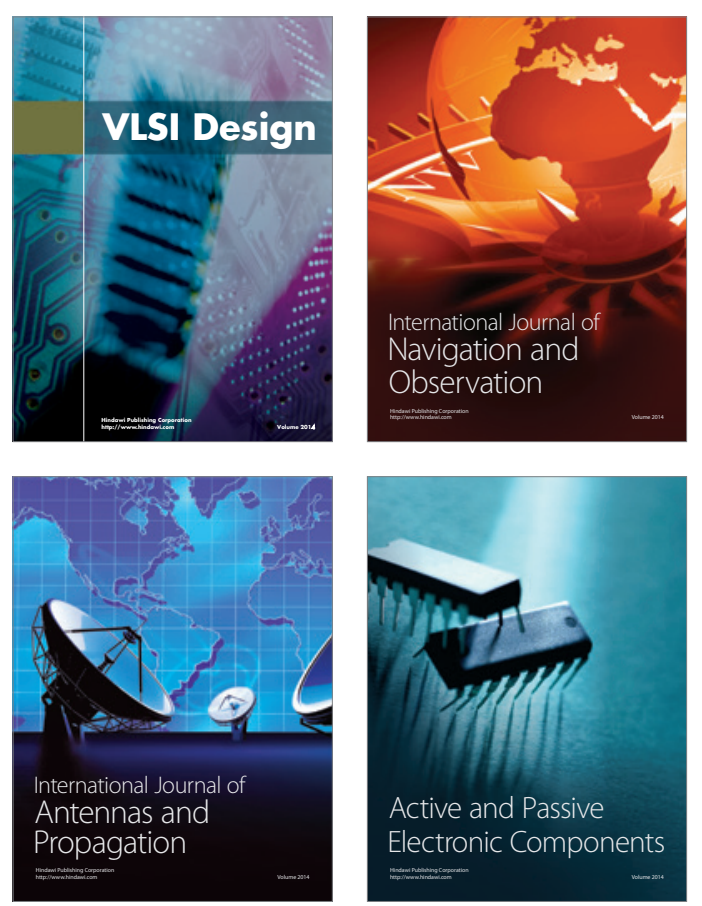
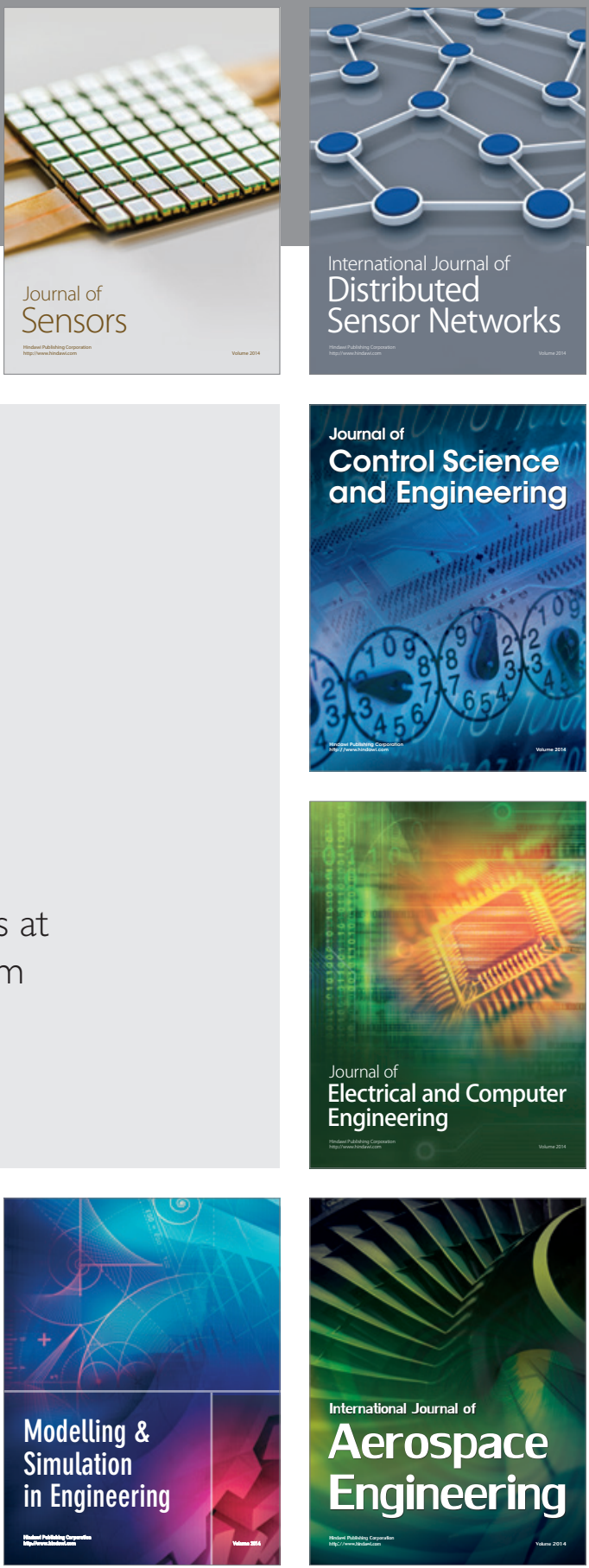

Journal of

Control Science

and Engineering
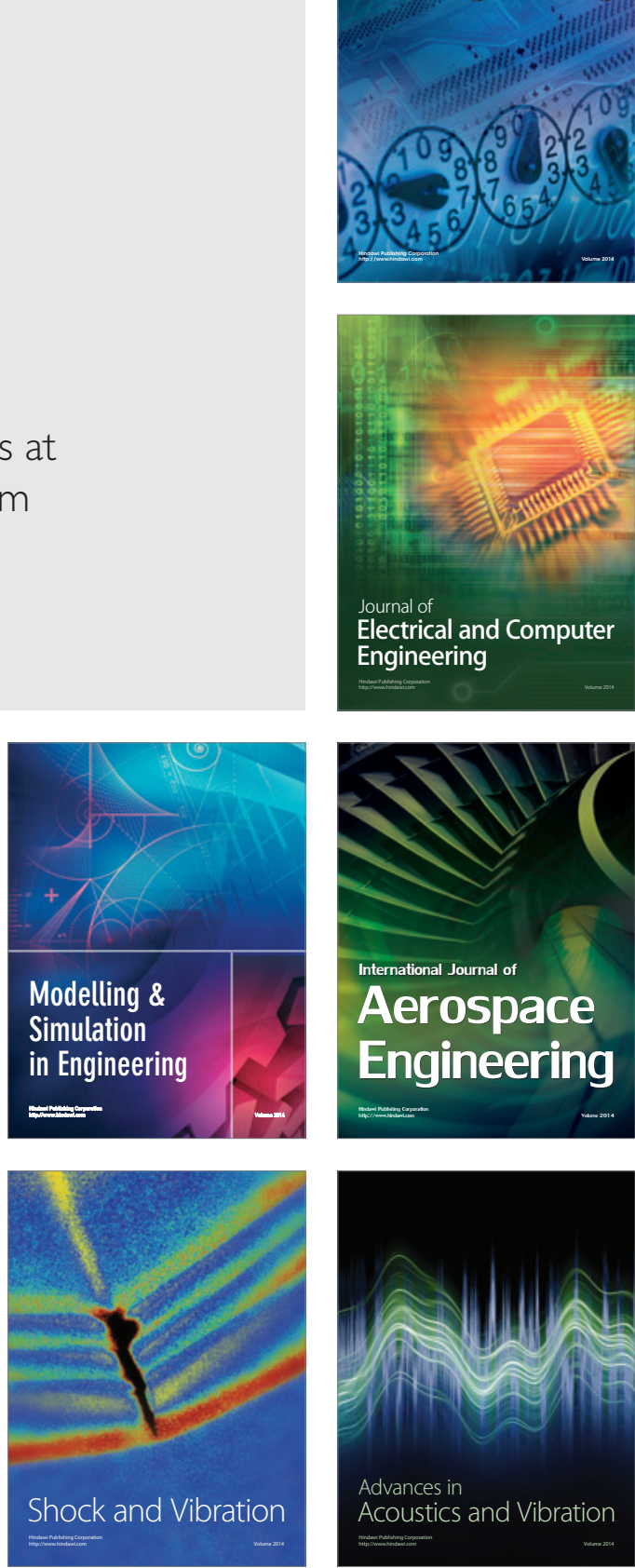